\title{
Angular dependent planar metamagnetism in the hexagonal compounds TbPtIn and TmAgGe
}

\author{
E. Morosan, S. L. Bud'ko and P. C. Canfield \\ Ames Laboratory and Department of Physics and Astronomy, \\ Iowa State University, Ames, IA 50011, USA
}

(Dated: July 30, 2021)

\begin{abstract}
Detailed magnetization measurements, $\mathrm{M}(\mathrm{T}, \mathrm{H}, \theta)$, were performed on single crystals of TbPtIn and TmAgGe (both members of the hexagonal $\mathrm{Fe}_{2} \mathrm{P} / \mathrm{ZrNiAl}$ structure type), for the magnetic field $\mathrm{H}$ applied perpendicular to the crystallographic c axis. These data allowed us to identify, for each compound, the easy-axes for the magnetization, which coincided with high symmetry directions ([120] for TbPtIn and [110] for TmAgGe). For fixed orientations of the field along each of the two six-fold symmetry axes, a number of magnetically ordered phases is being revealed by $\mathrm{M}(\mathrm{H}, \mathrm{T})$ measurements below $T_{N}$. Moreover, $T \simeq 2 \mathrm{~K},\left.\mathrm{M}(\mathrm{H})\right|_{\theta}$ measurements for both compounds (with $\mathrm{H}$ applied parallel to the basal plane), as well as $T=20 \mathrm{~K}$ data for TbPtIn, reveal five metamagnetic transitions with simple angular dependencies: $H_{c i, j} \sim 1 / \cos (\theta \pm \varphi)$, where $\varphi=0^{\circ}$ or $60^{\circ}$. The high field magnetization state varies with $\theta$ like $2 / 3 * \mu_{\text {sat }}\left(\mathrm{R}^{3+}\right) * \cos \theta$, and corresponds to a crystal field limited saturated paramagnetic, CL-SPM, state. Analysis of these data allowed us to model the angular dependence of the locally saturated magnetizations $M_{s a t}$ and critical fields $H_{c}$ with a three coplanar Ising-like model, in which the magnetic moments are assumed to be parallel to three adjacent easy axes. Furthermore, net distributions of moments were inferred based on the measured data and the proposed model.

PACS numbers: 75.25.+z; 75.10.-b; 75.30.Gw; 75.30.Kz; 75.50.Ee
\end{abstract}

\section{INTRODUCTION}

Numerous studies of angular dependent local moment metamagnetism show that simple geometric relationships exist between the critical fields of the metamagnetic phase transitions, and the angle that the applied field makes with the corresponding easy axis. One simple case is that of $\mathrm{TbNi}_{2} \mathrm{Ge}_{2} \frac{1}{2}$, a tetragonal compound with $\mathrm{Tb}$ ions in tetragonal point symmetry, where, at low temperatures, the crystalline electric field (CEF) anisotropy confines the local moments along the $c([001])$ crystallographic axis (Ising-like system). Consequently, several metamagnetic transitions are observed, with the critical field values $H_{c}$ having a $1 / \cos \theta$ dependence on the angle between the applied field $H$ and the c-axis. A more complex situation is encountered when the magnetic moments are allowed more degrees of freedom, i.e. when the CEF anisotropy constrains them to an easy plane. This is the case in the tetragonal compounds $\mathrm{RNi}_{2} \mathrm{~B}_{2} \mathrm{C}^{2.3 .4}$, with $\mathrm{R}=\mathrm{Tb}-\mathrm{Er}$, and $\mathrm{RAgSb}_{2}{ }^{5}$ for $\mathrm{R}=\mathrm{Dy}$. The $\mathrm{R}$ ions are again in tetragonal point symmetry and the local moments are confined to four equivalent [110] or [100] crystallographic directions; thus the angular dependent magnetization measurements, when the field is applied in the basal plane, reveal the four-fold anisotropy of the longitudinal magnetization that reflects the symmetry of the unit cell. The angular dependencies of the locally saturated magnetizations $M_{\text {sat }}$ and the critical fields $H_{c}$ could be treated by simple analysis, and plausible net distribution of moments could be inferred for each metamagnetic phase ${ }^{3.5}$. Kalatsky and Pokrovsky ${ }^{6}$ elaborated the four-position clock model, which agrees well with the observed metamagnetism in $\mathrm{HoNi}_{2} \mathrm{~B}_{2} \mathrm{C}$ and $\mathrm{DyAgSb}_{2}$.
Initially thought to be improbable, angular dependent metamagnetism in extremely planar systems is now an accepted and understandable event in tetragonal compounds; but this has not yet been well studied in hexagonal compounds, and this is the motivation for the present analysis. Recently we reported the presence of metamagnetism in a hexagonal class of materials, i.e. the RAgGe compounds, for $\mathrm{R}=\mathrm{Tb}-\mathrm{Tm}^{7}$. They crystallize in the ZrNiAl-type structure, an ordered variant of the hexagonal $\mathrm{Fe}_{2} \mathrm{P}$ family. In this structure, there is a unique rare earth site in the unit cell, with the rare earth ions occupying equivalent $3 \mathrm{~g}$ positions with orthorhombic point symmetry (fig.1). The anisotropic susceptibility was found to be axial for TbAgGe and progressed towards extremely planar for TmAgGe. The physical properties of the TmAgGe compound (antiferromagnetic ordering in the ground state, extremely planar anisotropy, metamagnetism when the applied field was perpendicular to the c-axis) are very similar to those of the isostructural TbPtIn system, previously only known in polycrystalline form ${ }^{8,9}$, making both compounds good candidates for a study of the angular dependent metamagnetism in hexagonal systems. In both TmAgGe and TbPtIn the $\mathrm{R}$ ions occupy the same site, whereas both ligands are different. Having two such systems will allow us to show that the behavior we find is not specific to one compound, but a more general result associated with this structure, or perhaps with the orthorhombic point symmetry in a hexagonal unit cell. As we shall see, the Néel temperature is much higher for TbPtIn $(46.0 \mathrm{~K})$ than for TmAgGe (4.2 K), with the former also showing a possible spin reorientation transition at a fairly high temperature $\left(T_{m}=27.4 \mathrm{~K}\right)$. This latter transition in TbPtIn was 
missed by the measurements on polycrystalline samples ${ }^{9}$, where even the nature of the magnetically ordered state below $\sim 50 \mathrm{~K}$ was not identified. Thus we can once again emphasize the advantage of analysis on single crystals rather than on polycrystalline samples.

This paper is organized as follows: after a brief description of the experimental methods, we present the $M(T, H)$ data on TbPtIn, emphasizing the complexity of its ordered state, for field orientations along the two in-plane, high symmetry directions; this is followed by the $\mathrm{M}(\mathrm{H}, \theta)$ measurements at low temperature, from which the values of the locally saturated magnetizations and critical fields as a function of $\theta$ were extracted. Next, we will introduce a model for the net distribution of moments, which we subsequently use to calculate the expected, locally saturated magnetizations $M_{j}$ and the critical fields (for the transition from state $\mathrm{i}$ to state j) $H_{c i, j}$ as functions of $\theta$. This will be followed by a comparison of how the calculated and experimental $M_{j}$ and $H_{c i, j}$ values vary with the angle between the applied field $\mathrm{H}$ and the easy axis for this compound.

Similar measurements performed on TmAgGe will then be shown, leading to the corresponding experimental $M_{j}(\theta)$ and $H_{c i, j}(\theta)$ phase diagrams, which we will again map onto the appropriate model calculation.

We will also analyze similar $M(H, \theta)$ data of TbPtIn for $T=20 \mathrm{~K}$, and use the same model in order to characterize a magnetic phase present only at higher temperatures.

Finally, we will summarize our key results and also indicate how our model can be generalized to a variety of possible point symmetries in tetragonal and hexagonal systems.

\section{EXPERIMENTAL METHODS}

Using an initial concentration of $\mathrm{Tb}_{0.05} \mathrm{Pt}_{0.05} \mathrm{In}_{0.90}$, clean hexagonal rods of TbPtIn were grown; this In-rich self-flux was used because of its low-melting temperature and because it introduces no new elements into the melt. The constituent elements were placed in alumina crucibles and sealed in quartz tubes under partial argon pressure; subsequently they were heated up to $1200^{\circ} \mathrm{C}$, and then cooled down to $800^{\circ} \mathrm{C}$ over $48 \mathrm{~h}$. Removal of the excess liquid solution revealed hexagonal rods with the c-axis along the axis of the rod. The crystal structure was confirmed by room temperature powder x-ray diffraction measurements, using $\mathrm{Cu} \mathrm{K}_{\alpha}$ radiation, from which the lattice parameters $\mathrm{a}=(7.55 \pm 0.01) \AA$ and $\mathrm{c}$ $=(3.88 \pm 0.01) \AA$ were also obtained. It is worth noting that the above temperature profile yielded in most cases well-formed, fairly good crystals (residual resistivity ratio RRR $=5.2$ ); however, some of the crystals had hollow channels in the center, sometimes with flux inclosures, and the quality of the resulting hexagonal rods seems to only deteriorate with slower cooling rates (i.e. $400^{\circ} \mathrm{C} /$ 100h). Single crystals of TmAgGe were also flux-grown out of Ag-Ge self flux, as described in detail in Morosan et al $\stackrel{7}{\underline{7}}$.

Magnetic measurements were performed in a Quantum Design Magnetic Properties Measurement System (MPMS) SQUID magnetometer, with a specially modified sample holder to allow the rotation of the sample so that its c-axis stays perpendicular to the applied magnetic field. Additional measurements up to $140 \mathrm{kG}$ were also taken, using an extraction magnetometer in a Quantum Design Physical Properties Measurement System (PPMS). In order to avoid torque on the rotator due to the extreme anisotropy of the samples, small mass samples (i.e. $\mathrm{m}=(0.40 \pm 0.05) \mathrm{mg}$ in the case of TbPtIn, and $\mathrm{m}=(0.54 \pm 0.05) \mathrm{mg}$ for TmAgGe) were used for the angular dependent measurements. To correct for the large weighing errors that would result from such small masses, we used a $13.55 \mathrm{mg}$ TbPtIn sample, and a $2.40 \mathrm{mg} \mathrm{TmAgGe}$ sample respectively, to measure $M(H)$ curves for $\theta=0^{0}$ and $30^{\circ}$ (with the angle $\theta$ measured between the applied field $\mathrm{H}$ and the easy axis, as described below). Whereas some errors are still introduced by this calibration due to the manual orientation of the large samples, the angular accuracy with which we are able to orient them is probably within $10^{\circ}$. The various $M(H, \theta)$ measurements on the smaller samples were than calibrated using the data for the larger mass pieces; this is believed to be very accurate given that the angular uncertainty in the rotator is less than $1^{0}$. There is additional uncertainty introduced by possible misalignment of the small piece with the c-axis exactly perpendicular to the applied field (due to the construction of the sample holder, this misalignment shouldn't be more than $10^{\circ}$ ).

Laue experiments have been performed on well-formed single crystals. They confirmed that the as-grown facettes of the hexagonal rods are alternatingly parallel to the (101) and (011) crystallographic planes (the macroscopic facettes are aligned with the hexagonal unit cell axes [100] or [010]).

\section{TbPtIn}

Fig.2 shows the inverse magnetic susceptibility for TbPtIn, as well as the low-temperature magnetization in the inset. This compound appears to order antiferromagnetically below $T_{N}=46.0 \mathrm{~K}$, with what is probably a spin-reorientation or a commensurate-toincommensurate transition around $T_{m}=27.4 \mathrm{~K}$, as indicated by the peaks in the $d(M * T / H) / d T$ plot $^{10}$ in Fig.3a. In the determination of these temperature values, $M / H$ represents the polycrystalline average susceptibility $\chi_{\text {ave }}$, calculated as

$\chi_{\text {ave }}=\frac{1}{3} \chi_{c}+\frac{2}{3} \chi_{a b}$

or, when measurements have been done along all three salient directions,

$\chi_{\text {ave }}=\frac{1}{3}\left(\chi_{[001]}+\chi_{[100]}+\chi_{[120]}\right)$.

The above temperatures are further confirmed by the $C_{p}(T)$ and $\rho(T)(i \| a b)$ data, also shown in Fig.3b and c. 
In the ordered state, as well as in the paramagnetic state up to $\sim 100 \mathrm{~K}$, the susceptibility is extremely anisotropic, with the local $\mathrm{Tb}$ moments confined to the $a b$-plane at low temperatures. To check the origin of the anisotropy in the paramagnetic state, single crystals of YPtIn were grown, with a small number of nonmagnetic $\mathrm{Y}^{3+}$ ions substituted with magnetic $\mathrm{Tb}^{3+}$ ions. The extremely anisotropic susceptibility and magnetization of the diluted compound demonstrate that this is single-ion anisotropy associated with the CEF splitting of the Hund's rule ground state $\mathrm{J}$ multiplet. From the Curie-Weiss effective moment, as determined from the average inverse susceptibility (Fig.4), the concentration $\mathrm{x}$ of the diluted compound $\left(\mathrm{Tb}_{x} \mathrm{Y}_{1-x}\right) \mathrm{PtIn}$ is $x=0.024$, whereas the high field magnetization equals $\sim 5.6 \mu_{B} / \mathrm{Tb}^{3+}$. (If we assume the high field magnetization to be $\sim 6.25 \mu_{B} / \mathrm{Tb}^{3+}$, as expected based on the model described below, the resulting concentration will be $x=0.019$ ).

For temperatures higher than $150 \mathrm{~K}$, Curie-Weiss behavior of the pure TbPtIn compound can be inferred (Fig.2) from the linear inverse susceptibilities, resulting in anisotropic Weiss temperatures $\Theta_{a b}=39.0 \mathrm{~K}$ and $\Theta_{c}=23.3 \mathrm{~K}$. The polycrystalline average susceptibility $\chi_{\text {ave }}$ yielded an effective moment $\mu_{\text {eff }}=9.74 \mu_{B} / \mathrm{Tb}^{3+}$, very close to the theoretical value $9.72 \mu_{B}$; the corresponding Weiss temperature is $\Theta_{\text {ave }}=33.3 \mathrm{~K}$.

The field dependent magnetization measurements shown in Fig.5a not only confirm the in-plane/out-ofplane anisotropy observed in the ordered state, but also indicate anisotropic magnetization within the basal plane. Moreover, several metamagnetic transitions can be seen for the field parallel to the $a b$-plane, for fields up to $140 \mathrm{kG}$ in the $M(H)$ data, and for the magnetoresistance measurements up to $90 \mathrm{kG}$ in Fig.5b. The geometry of the crystals led to more uncertainty in orienting the resistance pieces than the ones for magnetization measurements; therefore we can infer the approximate orientation of the magnetoresistance sample with respect to the applied field, by comparing the $\Delta \rho(H) / \rho(0)$ data (Fig.5b) with the $M(H)$ curves for $H \| a b$ (Fig.5a): since various features in the magnetoresistance measurements occur closer to the critical fields in $M([120])$, we can assume that the field was almost parallel to the [120] direction. (The sharp drop in $\rho(H)$ below $\sim 1 \mathrm{kG}$ (Fig.5b) is very likely due to superconductivity of residual In flux on the surface of our resistance bar). For $H$ along the c-axis, the magnetization increases almost linearly with increasing field (Fig.5a), while staying far smaller than $M_{a b}$.

At the highest applied field ( $H=140 \mathrm{kG})$, the magnetization values for the three shown orientations are $M([110])=5.86 \mu_{B} / \mathrm{Tb}, M([120])=6.45 \mu_{B} / \mathrm{Tb}$ and $M([001])=0.92 \mu_{B} / \mathrm{Tb}$. Whereas the extreme planar anisotropy of TbPtIn and the anisotropy within the $a b$-plane recommended this compound for a study of the angular dependent metamagnetism, the fact that the magnetization values were smaller in all three direc- tions than the calculated $\mu_{\text {sat }}=9 \mu_{B}$ for $T b^{3+}$ ions is somewhat intriguing. One plausible explanation for the low magnetization values would be the existence of more metamagnetic transitions for fields unaccessible with our measurement systems (i.e. above $140 \mathrm{kG}$ ). Another possibility is that an additional energy scale (such as CEF splitting) exists, that confines the three local moments to three distinct, non-collinear, in-plane orientations. As shall be shown below, we believe the latter to be the more likely scenario.

In order to determine the easy axes of the system, we continuously rotated a small piece of the diluted sample $\left(\operatorname{Tb}_{x} \mathrm{Y}_{1-x}\right) \operatorname{PtIn}(x \approx 0.02)$ in an applied field $H=55 \mathrm{kG}$ (perpendicular to $c$ ); for constant temperature $T=2 \mathrm{~K}$, the corresponding magnetization measurement, shown as open symbols in Fig.6, roughly follows a $\cos \theta$ dependence (solid line) around the closest [120]-equivalent directions (i.e. $\theta=60^{\circ} * n$, $n=$ integer), where the maxima occur. As we have seen before, the concentration of the diluted sample is $x=(0.0215 \pm 0.0025)$; given this uncertainty, the absolute value of the magnetization $M(\theta)$ in Fig.6 could not be determined, and thus we scaled the data to the maximum value, $M\left(\theta=0^{0}\right)$. Similar behavior appears in the pure TbPtIn compound (full symbols in Fig.6), where the measured data have also been scaled to their corresponding maximum value at $\theta=0^{0}$. We can conclude that the easy axes of the TbPtIn system are the [120] directions. However dramatic departures from the $\cos \theta$ angular dependence can be noticed. The magnetization for TbPtIn, indicative of strong interactions between the local moments, is also consistent with various metamagnetic states crossing the $H=55 \mathrm{kG}$ line at different angles. Based on the above data, we will consider the easy axes to be the [120]-equivalent directions, and the angle $\theta$ will be measured from the closest easy axis.

In order to get an idea about the various metamagnetic states in this compound, we first explored changes of the critical fields and temperatures for two fixed orientations. The corresponding $\left.M(T)\right|_{H, \theta}$ and $\left.M(H)\right|_{T, \theta}$ measurements shown in Fig.7-8 have been used to determine the $H-T$ phase diagrams for the two in-plane high symmetry directions, $H \|[120]$ and $H \|[110]$ respectively. As illustrated in the insets in these figures, the points in these phase diagrams have been determined from local maxima in $d(M * T) / d T$ (full circles in fig.9) for fixed fields, and in $d M / d H$ (open circles in fig.9) for various $M(H)$ isotherms. Even though in Fisher et al ${ }^{10}$ the maxima in $d(M * T) / d T$ criterion is described only for antiferromagnetic systems, we apply it here not only for the AF state, but also for high-field states, where the magnetization has a net ferromagnetic component. We are confident that small errors are thus introduced, given the consistency of the critical field and temperature values obtained from both $d(M * T) / d T$ and $d M / d H$ derivatives (full and open symbols respectively in Fig.9). Given that the transition peaks were broad for some field and temperature values, we used Lorentzian fits of the corresponding derivatives 
(thick lines in fig.7 and 8, insets) to determine the critical values $H_{c}$ and $T_{c}$.

The resulting $H-T$ phase diagrams for $H \|[120]$ (fig.9a) and $H \|[110]$ (fig.9c) are qualitatively similar, at low temperatures and low fields showing the metamagnetic states already seen in the $M(H)$ data in Fig.5. For $H \|[120]$, fig.9a shows that the antiferromagnetic ground state persists up to about $20 \mathrm{kG}$, followed by a small intermediate state $M_{1}$ (between $\sim 20 \mathrm{kG}$ and $28 \mathrm{kG}$ ) and a higher field state $M_{2}$ up to $\sim 54 \mathrm{kG}$; as field is being further increased, the paramagnetic $P M$ state is reached, as already indicated in fig.5a by the horizontal plateaux measured up to $140 \mathrm{kG}$. (At low temperatures, this is a crystal-field limited saturated paramagnetic CLSPM state, in which, as discussed below, all moments are assumed to be in their 'up' positions, while still confined by the strong CEF energy to three distinct, non-collinear directions within the basal plane.) When moving up in temperature at low fields, we find the antiferromagnetic ground state to extend up to $\sim 27.4 \mathrm{~K}$, whereas the magnetic ordered state persists up to $\sim 46.0 \mathrm{~K}$; both transition temperatures have been already observed (fig.2 and $3)$. The $M_{1}$ phase exists below $\sim 5.0 \mathrm{~K}$, after which, for a limited temperature range $(5.0 \mathrm{~K}<T<15.0 \mathrm{~K})$, there is a direct transition from the $A F$ to the $M_{2}$ state. Between $15.0 \mathrm{~K}$ and $27.4 \mathrm{~K}$, or $18.4 \mathrm{kG}$ and $2.0 \mathrm{kG}$ respectively, another intermediate phase, $M_{4}$, forms. The inset in fig. 8 a represents an example of two isothermal cuts of the [120] phase diagram in fig.9a: the phase boundaries of the bubble-like phase $M_{4}$ are very close in field at constant $\mathrm{T}$, and so the lower peak in the $T=20.0 \mathrm{~K}$ isothermal derivative $d M / d H$ is poorly defined; the two higher peaks are fairly sharp, similar to the one defining the $M_{2}$ to $P M$ transition in the $T=30.0 \mathrm{~K}$ isotherm. However, as we move down in field along the latter isotherm, one broad peak around $15.4 \mathrm{~K}$ may indicate the crossing of another almost horizontal phase boundary (leading into the $M_{5}$ state), and thus hard to identify in $d(M * T) / d T$. One more peak at $H_{c} \simeq 3.4 \mathrm{kG}$ is indicative of possibly another phase $A F^{\prime}$ existing below this field, between $28 \mathrm{~K}$ and $46.0 \mathrm{~K}$. This is consistent with an antiferromagnetic ordered state below $46.0 \mathrm{~K}$, with an incommensuratecommensurate transition around $28 \mathrm{~K}$ which frequently occurs in intermetallic compounds.

For $H \|[110]$ (Fig.9c) the $H-T$ phase diagram is fairly similar, with only a few differences: a far less distinct $M_{1}$ phase and a lower upper-boundary for the $M_{2}$ region. However, the most notable difference is a new high field phase, $M_{3}$, whose upper boundary is determined by the points indicated with small arrows in fig.7b. (As the field is being increased towards $140 \mathrm{kG}$, this line becomes almost vertical, making it difficult to identify also in $\left.M(H)\right|_{T}$ measurements.)

In order to see how the $H-T$ phase diagram evolves from $H \|[120]$ to $H \|[110]$, we collected comparable $\left.M(T)\right|_{H}$ and $\left.M(H)\right|_{T}$ data for an intermediate orientation of the applied field (approximately $12^{0}$ from the easy axis [120]). Fig.10a shows these $M(T)$ curves for
$H=1-70 \mathrm{kG}$, with the small arrows indicating the highest-T transition at each field value, as determined from the $d(M * T) / d T$ maxima. Thus, the upper-most phase boundary in Fig.9b, representing the phase diagram for this intermediate position, can be followed in field up to $H=65 \mathrm{kG}$ (full symbols). For this orientation, we can also identify this line in the $M(H)$ data, and an example is shown in Fig.10b for the $T=10 \mathrm{~K}$ $M(H)$ isotherm and its $d M / d H$ derivative. Overall, the features common to both Fig.9a and c are also present in Fig.9b; moreover, in going from the [110] to the [120] direction, the $M_{3}$ phase is being compressed, such that in the intermediate position the phase boundary separating it from $P M$ is fully delineated below $70 \mathrm{kG}$.

Given the clear in-plane anisotropy of the magnetization (fig.5-10), it becomes desirable to systematically determine the angular dependence of the critical fields $H_{c i, j}(\theta)$ and locally saturated magnetizations $M_{j}(\theta)$ of the $H \perp c$ metamagnetic transitions at $T=2 \mathrm{~K}$.

Fig.11 shows a series of magnetization isotherms $(T=2 \mathrm{~K})$ measured at various angles relative to the easy axis. The critical fields $H_{c i, j}$, for the transition between states $\mathrm{i}$ and $\mathrm{j}$, were determined from maxima in $d M / d H$, as exemplified in fig.12, and are shown as full symbols in Fig.13a. In most cases, Lorentzian fits of the derivative peaks were used (solid line in Fig.12) to more accurately determine the critical field values. The open symbols in fig.13 represent reflections of the measured data across the $\theta=0^{0}$ direction; whenever the measured points extend beyond the $0^{0} . .30^{0}$ region, they almost coincide with the calculated reflections, as expected for a symmetry direction.

For $\theta \leq 12^{0}$ the antiferromagnetic AF ground state exists for fields up to about $20 \mathrm{kG}$, after which two closely spaced metamagnetic transitions occur, with critical fields, at $\theta=0^{0}, H_{C A F, 1}=20.5 \mathrm{kG}$ (for the $\mathrm{AF}$ to $M_{1}$ transition) and $H_{c 1,2}=27.7 \mathrm{kG}$ (corresponding to the transition from the $M_{1}$ to the $M_{2}$ state). A third transition from $M_{2}$ to $C L-S P M$, around a critical field $H_{c 2, C L-S P M}=53.7 \mathrm{kG}$, changes very little with angle up to $\theta \approx 8^{0}$; for higher angles, another metamagnetic state $M_{3}$ forms, being delineated by two distinct critical fields, $H_{c 2,3}$ and $H_{c 3, C L-S P M}$. As the former decreases with the angle, the latter soon reaches values around the maximum field of $55 \mathrm{kG}$ available in the SQUID magnetometer used for these measurements. In order to follow this latest transition in higher magnetic fields, additional measurements were taken in a different magnetometer, for fields up to $70 \mathrm{kG}$, and a slightly different temperature $(T=1.85 \mathrm{~K})$; these data are shown in the inset in Fig.11a, but by $\theta=12^{0}, H_{c 3, C L-S P M}$ becomes larger than $70 \mathrm{kG}$, therefore we can only anticipate that this transition still exists for larger angles. (The $H-T$ phase diagrams (Fig.9b and c) seem to indicate that this critical field value increases from $\sim 68 \mathrm{kG}$ for $\theta \cong 12^{0}$, to more than $140 \mathrm{kG}$ at $\theta=30^{\circ}$ ). Slight differences can be noticed between the data sets taken in the two machines, very likely due to the different temperatures at 
which they were taken. A linear scaling of the two data sets by a factor of $\sim 1.07$ was necessary, for both the magnetization values and the critical fields; the scaling of the magnetization values can be explained by an assumption of slightly different angles between the applied field and the rotator axis in each magnetometer, while the field values may have changed with $\mathrm{T}$ according to the phase diagrams in Fig.9.

After the scaling of the two data sets, and after additional calibration to the measurements on the large mass $13.55 \mathrm{mg}$ piece, the locally saturated magnetization values were determined. The criterium used for determining the magnetization for each state $\left(M_{j}\right)$, was the onset $M(H)$ value (fig.12), i.e. the intersection of the linear fit of the $M_{j 2}$ magnetization plateau and the highestslope linear fit of the $M(H)$ curve during the $M_{j_{1}}$ to $M_{j_{2}}$ transition. More attention was given to determining the magnetization for the first state $\left(M_{1}\right)$, due to the limited field range over which this state exists. Several criteria tried in this case (onset value, midpoint between transitions, minimum in $d M / d H$ or midpoint on the appropriate linear region on the $M(H)$ curves) resulted in almost identical angular dependencies of $M_{1}$; moreover, using any of the aforementioned criteria, we were still unable to follow this state in the $M(H)$ curves for angles beyond $25^{0}$.

For $\theta>12^{0}$, the similar two sets of measurements are shown in Fig.11b. The same criteria were used for the determination of $M_{j}(\theta)$ and $H_{c i, j}(\theta)$. The two lower metamagnetic transitions can also be seen in this region, while of the higher two, only $H_{c 2,3}$ is within our field range; as the angle increases, the first two transitions move closer in field $\left(H_{c A F, 1}\right.$ increases, while $H_{c 1,2}$ doesn't vary significantly with $\theta$ ), such that the $M_{1}$ state becomes very narrow, making its determination very difficult. $M_{2}$ and $M_{3}$ however appear as well defined plateaus, continuously decreasing, and increasing respectively, from the local extremum values seen at $\theta=0^{0}$. $H_{c 2,3}$ has a minimum of $38.9 \mathrm{kG}$ around $\theta=30^{\circ}$.

The magnetization curves revealed four metamagnetic states, and their angular dependence is presented in Fig.13b: $M_{1}, M_{2}$ and $M_{C L-S P M}$, which have local maxima at $\theta=0^{0}$ around $1.08 \mu_{B} / \mathrm{Tb}, 3.00 \mu_{B} / \mathrm{Tb}$, and $6.25 \mu_{B} / \mathrm{Tb}$ respectively, and $M_{3}$ which exists only beyond $\theta=8^{0}$ and has a maximum of $5.06 \mu_{B} / \mathrm{Tb}$ at $\theta=30^{0}$. Similar to the $H_{c i, j}$ in Fig.13a, the open symbols in Fig.13b represent reflections of the measured data across the $\theta=0^{0}$ (easy axis) direction.

The dotted lines in Fig.13a and $\mathrm{b}$ are fits to $H_{c i, j}(\theta)$ and $M_{j}(\theta)$ respectively, as calculated based on the model that will be discussed below. Their angular dependencies are described by $1 / \cos \left(\theta \pm \varphi_{j}\right)$, and $\cos \left(\theta \pm \varphi_{j}\right)$ respectively, with $\varphi_{j}=0^{0}, 30^{0}$ or $60^{\circ}$. These values are integer or half-integer multiples of $360^{\circ} / n$, where $n=6$ in our hexagonal system. Considering the sixfold symmetry of the this compound, these simple geometrical relationships render TbPtIn as very similar to $\mathrm{RNi}_{2} \mathrm{~B}_{2} \mathrm{C}^{3.4}$ or DyAgSb${ }_{2}{ }^{\frac{5}{2}}$, tetragonal compounds where the analogues $\varphi_{j}$ values were $0^{0}, 45^{0}$ or $90^{0}$ (integer or half-integer multiples of $360^{\circ} / n$, where $n=4$ ).

As seen earlier in the cases of the tetragonal compounds $\mathrm{HoNi}_{2} \mathrm{~B}_{2} \mathrm{C}^{3}$ or DyAgSb${ }_{2}{ }^{5}$, simple angular dependencies of the critical fields, as well as of the locally saturated magnetizations exist in the hexagonal compound TbPtIn; this will be further confirmed by similar geometrical relationships that appear to exist in TmAgGe.

\section{DATA ANALYSIS}

The field and temperature dependent magnetization measurements on TbPtIn (fig.2 and 5) have shown that this compound is extremely anisotropic, with the magnetic moments confined to the hexagonal basal plane. Moreover, when the direction of the applied field is varied within the basal plane, six fold anisotropy of the saturated magnetization is revealed in both TbPtIn and its dilution $\left(\mathrm{Tb}_{x} \mathrm{Y}_{1-x}\right) \mathrm{PtIn}$ (Fig.6). Consequently, detailed magnetization measurements with $H \perp c$ were performed, allowing us to quantitatively describe the angular dependencies of the critical fields $H_{c i, j}$ and locally saturated magnetizations $M_{j}$ (Fig.13). By analogy to the fourposition clock mode ${ }^{3,5.6}$ for tetragonal systems, we are now proposing a simple model for the net distribution of moments in the hexagonal compound TbPtIn: three coplanar Ising-like systems, $60^{\circ}$ apart in the basal plane. Such a hypothesis was first suggested by the high field magnetization values observed in the pure compound TbPtIn, as well as in the highly diluted $\left(\mathrm{Tb}_{x} \mathrm{Y}_{1-x}\right)$ PtIn. As the maximum measured magnetization for TbPtIn was around $6 \mu_{B} / \mathrm{Tb}^{3+}$ (far smaller than the calculated $9 \mu_{B}$ value), it is reasonable to assume the existence of more metamagnetic transitions beyond our maximum applied field $H=140 \mathrm{kG}$. However, in the highly diluted compound, where, within our field and temperature ranges, we are only probing the paramagnetic state, the magnetization also reaches only $\sim 6 \mu_{B} / \mathrm{Tb}^{3+}$ at the highest $\mathrm{H}$. This is consistent with the $M=6 \mu_{B} / \mathrm{Tb}^{3+}$ corresponding to a crystal-field limited saturated paramagnetic (CL-SPM) state. Consequently we chose our model based on three Ising-systems such that it described the hexagonal symmetry of the compound having three magnetic ions in orthorhombic point symmetry, with the above value corresponding to saturation (in the limit of high CEF energy). In order to verify this hypothesis, the expected angular dependencies of possible moment configurations resulting from such a model will be compared with our measurements. Furthermore, we will use our experimental results to refine the model, by considering multiples of the three Ising-like systems, resulting in more complex angular dependencies of the calculated magnetization and critical field values.

In the $P \overline{6} 2 m$ space group, TbPtIn assumes a hexagonal crystal structure, with $3 \mathrm{~Tb}^{3+}$ ions at equivalent $3 \mathrm{~g}$ (orthorhombic) sites. The fact that a strong CEF anisotropy confines the local moments to the basal plane 
calls for a two dimensional model, greatly simplifying the analysis. (A schematic description of an equivalent three-dimensional model has been introduced for DyAgGe ${ }^{7}$ ). Having three equivalent magnetic moments in orthorhombic point symmetry, one possible way to achieve the overall hexagonal symmetry is by restricting the moments to three of the six-fold symmetry axes, $60^{\circ}$ apart, while allowing for both the 'up' (solid arrows) and 'down' (dotted arrows) positions for a given direction (Fig.14). Any specific Tb-site would, at low temperatures, behave like an Ising system, with each third of the sites having parallel Ising directions. Thus, each metamagnetic state of TbPtIn can be described by a multiple $\mathrm{S}$ of three Ising-like systems along three [120] equivalent directions (the easy axes for this system). We will use $\nwarrow$ ,$\uparrow$ and $\nearrow$ symbols, to denote the orientation of the three moments in their 'up' positions, and $\searrow, \downarrow$ and $\swarrow$ symbols respectively, for the corresponding 'down' positions. The order of the arrows is not meaningful for our model; only the number of arrows for each orientation is significant for the net distribution of moments. Moreover, we describe each metamagnetic state with the minimum-S value moment configuration consistent with the experimental data. However, higher $\mathrm{S}$ values are possible for most of the states, and information about the wave vectors (e.g. from scattering experiments) would be required to determine unique $\mathrm{S}$ values.

Since in our experiments we only measure the projection of the magnetic moment along the field direction, the angular dependence of the magnetization $M_{j}$ per moment of an arbitrary $3 \mathrm{~S}$-moments configuration is

$$
M_{j}(\theta) / \mu_{\text {sat }}\left(\mathrm{Tb}^{3+}\right)=\frac{1}{3 * S}\left[\sum_{i=1}^{S} m_{i} * \cos \left(\theta-60^{0}\right)+\right.
$$
$\left.\sum_{i=1}^{S} m_{i} * \cos \theta+\sum_{i=1}^{S} m_{i} * \cos \left(\theta+60^{\circ}\right)\right]$

where $\theta$ is a continuous variable representing the angle between the applied field and the closest easy axis $\left(-30^{0} \leq \theta \leq 30^{0}\right)$, and the three sums give the magnetization value due to each of the three directions of the Ising-like systems; the $m_{i}$ parameters equal \pm 1 , depending on whether a certain moment is in the 'up' $(+1)$ or 'down' $(-1)$ position for the respective direction. We restrict our model description to the $0^{0} \leq \theta \leq 30^{0}$ angular region, which, by symmetry across the $\theta=0^{0}$ direction, also describes the $-30^{0} \leq \theta \leq 0^{0}$ region.

For $S=1$, our model corresponds to one set of three such Ising-like systems. We assume that in high applied fields, the three magnetic moments occupy the three allowed easy axes closest to the direction of the field; as the field is lowered, the metamagnetic transitions occur such that the measured magnetization is being decreased with $\mathrm{H}$. In this hypothesis, there are three distinct moment configurations for the system: $(\nwarrow \uparrow \nearrow)$ for the CL-SPM state, $(\searrow \uparrow \nearrow)$ for intermediate field values, and $(\searrow \uparrow \swarrow)$ for the AF ground state. The above formula yields the following angular dependencies of the resulting longitudinal (measured) magnetizations: $2 / 3 * \cos \theta$, $2 / 3 * \cos \left(\theta-60^{\circ}\right)$ and 0 respectively, represented by open circles in Fig.15.

Fig.13b shows that such a model only describes the
CL-SPM state $M_{C L-S P M}(\theta) / \mu_{\text {sat }}\left(\mathrm{Tb}^{3+}\right)=2 / 3 * \cos \theta$ and the AF ground state $M_{A F} / \mu_{\text {sat }}\left(\mathrm{Tb}^{3+}\right)=0$ of TbPtIn; according to the proposed model, the local moment configurations from which the above angular dependencies follow are $(\nwarrow \uparrow \nearrow)$ and $(\searrow \uparrow \swarrow)$ respectively. It is worth noting that the CL-SPM magnetization value, calculated based on the above moment configuration, is $6 \mu_{B}$, smaller than the measured $6.25 \mu_{B}$. One possible explanation is that with increasing field, the system is slowly approaching the CEF splitting energy. This is also consistent with the increasing plateaus in the high field magnetization data in Fig.5a; however, the extrapolation of these plateaus down to $H=0$ results in smaller values for the [120] direction $\left(6.13 \mu_{B}\right)$ and the [110] direction $\left(5.35 \mu_{B}\right)$, closer to the calculated values $\left(6.0 \mu_{B}\right.$ and $\sqrt{3} / 2 * 6 \mu_{B}=5.2 \mu_{B}$ respectively).

To characterize all the other observed metamagnetic states, larger S-values are needed, i.e. the local moment configurations are described by an integer multiple $S>1$ of sets of three Ising-like systems. Fig.15 (crosses) also shows all possible angular dependencies of the magnetizations resulting from such a generalized model when $S=2$. By comparison with the experimental data, it appears from Fig.13b that two more metamagnetic states can now be described with $S=2$ :

$$
M_{3}(\theta) / \mu_{\text {sat }}\left(\mathrm{Tb}^{3+}\right)=2 \sqrt{3} / 6 * \cos \left(\theta-30^{0}\right) \text { (moment }
$$
configuration $(\nwarrow \nwarrow \uparrow \uparrow \swarrow \nearrow))$,

$$
\text { and }
$$

$M_{2}(\theta) / \mu_{\text {sat }}\left(\mathrm{Tb}^{3+}\right)=2 / 6 * \cos \theta$ (moment configuration $(\searrow \nwarrow \uparrow \uparrow \swarrow \nearrow)$ ).

There is still one more metamagnetic state, $M_{1}$, which cannot be described within the $S=2$ model; however, for most of the angular range, its magnetization has an angular dependence consistent with: $M_{1}(\theta) / \mu_{\text {sat }}\left(\mathrm{Tb}^{3+}\right)=2 / 18 * \cos \theta$. The $\cos \theta$ dependence (i.e. $\cos$ is an even function) requires that the moment configuration be symmetric with respect to the $\theta=0^{0}$ direction. The simplest possibility is $(\nwarrow \uparrow \nearrow)$, for which the magnetization varies as $2 / 3 * \cos \theta$; to this, a number of sets of three moments needs to be added, with zero net magnetization (e.g. multiples of $(\searrow \uparrow \swarrow)$ or $(\searrow \nwarrow \uparrow \downarrow \swarrow \nearrow)$ ), to get a resulting magnetization amplitude of $2 / 18$. Consequently a minimum $S=6$ configuration $(\searrow \searrow \searrow \searrow \backslash \uparrow \uparrow \uparrow \uparrow \uparrow \uparrow \swarrow \swarrow \swarrow \swarrow \swarrow \nearrow)$ or $(\searrow \searrow \searrow \nwarrow \nwarrow \downarrow \downarrow \uparrow \uparrow \uparrow \uparrow \swarrow \swarrow \swarrow \nearrow \nearrow \nearrow)$ yields the desired calculated magnetization $2 / 18 * \cos \theta$.

Assuming the above net distributions of moments for the observed metamagnetic states, one can derive the expected angular dependencies of the critical fields. Comparison between the data in Fig.13a and these calculated $H_{c i, j}(\theta)$ values will further confirm or refute the net distributions of moments proposed above.

Since the energy associated with a magnetic moment $\vec{M}$ in an applied field $\vec{H}$ is $\vec{M} \cdot \vec{H}^{5}$, the corresponding energy difference $\Delta E_{j i}$ between metamagnetic states $\overrightarrow{M_{i}}$ and $\overrightarrow{M_{j}}$ is:

$$
\Delta E_{j i}=\vec{M}_{j} \cdot \vec{H}-\vec{M}_{i} \cdot \vec{H}
$$


If there is a critical energy $E_{c}=\Delta E_{j i}$ to be exceeded for a metamagnetic transition between states $i$ and $j$ to occur, than the critical field value is given by:

$H_{c i, j}=\frac{E_{c}}{M_{j}-M_{i}}$,

where $M_{j}$ and $M_{i}$ are the measured (projections along the field) respective magnetizations. The numerator in the above expression is angle and field independent, and the angular dependence of $H_{c i, j}$ follows only from the denominator. In other words,

$H_{c i, j} \sim \frac{1}{M_{j}-M_{i}}$.

Consequently, the expected critical field values, shown as dotted lines in Fig.13a, are:

$H_{C A F, 1}(\theta) \sim 1 / \cos \theta$,

$H_{c 1,2}(\theta) \sim 1 / \cos \theta$

$H_{c 2,3}(\theta) \sim 1 / \cos \left(\theta-60^{\circ}\right)$,

$H_{C 3, C L-S P M}(\theta) \sim 1 / \cos \left(\theta+60^{\circ}\right)$

and $H_{C 2, C L-S P M}(\theta) \sim 1 / \cos \theta$.

The reflections across the $\theta=0^{0}$ direction result from the above formulas, when substituting $\theta$ with $-\theta$; moreover, since $\cos$ is an even function, this is equivalent to a change in sign only for the $\varphi$ in the above expressions written as $1 / \cos (\theta-\varphi)$.

As described above and similar to the analogous study in the tetragonal compound $\mathrm{HoNi}_{2} \mathrm{~B}_{2} \mathrm{C}^{3}$, in most cases we used maxima in $d M / d H$, and not the on-set criterion, to determine the critical field values, because the magnetizations during the transition were not always linear; however, comparison with the calculated critical fields based on the above model is still appropriate, given that only small departures from linearity were encountered, mostly close to the bordering states $\left(M_{i}\right.$ and $\left.M_{j}\right)$. (The non-linear change of the magnetization with increasing field indicates that other factors (i.e. the demagnetization factor of the sample $\frac{11}{1}$, coexistence of more than two phases, non-linear superposition of the various states) may be responsible for the broadening of the transition).

Comparison of the measured critical fields and locally saturated magnetizations (Fig.13a,b full and open symbols) with the calculated values as described above (Fig.13a,b dotted lines) confirms, in most cases, the assumed local distribution of moments. However, the first metamagnetic state $M_{1}$ follows well the calculated $2 / 18 * \mu_{\text {sat }}\left(\mathrm{Tb}^{3+}\right) * \cos (\theta)$ dependence up to $\theta \approx 25^{0}$, after which it is difficult to determine it with reasonable accuracy. Despite the fact that both $H_{c A F, 1}$ and $H_{c 1,2}$ should depend on this magnetization value, the former follows the expected angular dependence fairly well, whereas the latter falls under the calculated $1 / \cos \theta$ curve. Even though at this point we don't have a rigorous calculation to support our assumption, we anticipate that the proximity of the two lowest transitions requires a calculation with more than two coexisting phases, which may render a better fit of the observed experimental data in this region. On the other hand, the $H_{c 2,3}$ and $H_{c 3, C L-S P M}$ critical fields, which have the most evident angular dependence, are well fitted by the calculated functions based on the present model. It is thus reasonable to assume that, whereas possibilities for refining the model exist, in the simple form that we present here it describes our system fairly well.

The polar plot in Fig.16 helps in understanding how the hexagonal crystal structure of this compound is reflected in the angular dependence of the metamagnetic phase transitions: similar to the polar phase diagrams for tetragonal compounds $\mathrm{HoNi}_{2} \mathrm{~B}_{2} \mathrm{C}^{\underline{6}}$ or $\mathrm{DyAgSb}_{2}{ }^{\frac{5}{2}}$, when we plot $H_{c} * \sin \theta$ vs. $H_{c} * \cos \theta$, the phase boundaries become straight lines, with slopes equal to either $\pm 1 / \sqrt{3}$ or $\infty$. These slopes correspond to directions either parallel or perpendicular to the high symmetry axes (i.e. [110] or [120]) in the hexagonal structure, just as within the four position clock model the corresponding phase lines were either parallel or perpendicular to the tetragonal high symmetry axes ([110] or [010]). As described in Myers et $a l_{\underline{5}}^{\underline{5}}$, the equations of these straight lines in polar coordinates can be used to verify the transitions already discussed: if we substitute the above slope values in the general formula

$$
\begin{aligned}
& R(\theta)=a /(\sin \theta-b * \cos \theta), \\
& \text { for a line with slope } b \text {, we get: } \\
& H_{c}(\theta) \sim 1 / \cos \theta \text { for } b=\infty \\
& H_{c}(\theta) \sim 1 / \cos \left(\theta-60^{0}\right) \text { for } b=-1 / \sqrt{3} \\
& \text { or } \\
& H_{c}(\theta) \sim 1 / \cos \left(\theta+60^{\circ}\right) \text { for } b=1 / \sqrt{3},
\end{aligned}
$$

which are consistent with the angular dependencies of the transitions determined above.

For the most part, the experimental points fall onto the calculated straight lines, as expected. Some deviations from the straight lines can be noticed, with the most evident one for $H_{c 1,2}$, for which we already emphasized the necessity of a more complex model. In a similar manner (even though only for fewer angles), $H_{c 2,3}$ curves under the calculated straight line as we move away from the [120] easy axis, while some even smaller deviations from linearity are apparent in $H_{C A F, 1}$; this may indicate that special attention needs to be paid in determining the angular dependence of the critical fields, when rotating from the proximity of one easy axis to another.

\section{TmAgGe}

We already reported the basic magnetic properties of $\mathrm{TmAgGe}{ }^{7}$, which strongly resemble those of TbPtIn: the magnetic susceptibility is extremely anisotropic (Fig.17), indicating antiferromagnetic order below $T_{N}=4.2 \mathrm{~K}$. The local magnetic moments are confined by the strong CEF anisotropy to the basal plane, both below and above $T_{N}$. This can also be seen in the field dependent magnetization measurements, shown in Fig.18, where in-plane anisotropy of the ordered state is also apparent. Similar to the case of TbPtIn, several metamagnetic transitions exist for both $H \|[110]$ and $H \|[120]$; these result in magnetization values of $4.92 \mu_{B}$ and $4.30 \mu_{B}$ respectively, at $H=70 \mathrm{kG}$, far below $\mu_{\text {sat }}\left(\mathrm{Tm}^{3+}\right)=7.0 \mu_{B}$, whereas for the $\mathrm{c}$ direction, the magnetization is linear and much smaller up to the maximum applied field. The ratio of the 
two in-plane magnetizations is $\frac{M([120])}{M([110])}=0.87$, close to the $\cos 30^{\circ}$ value expected within the model described before for TbPtIn for the CL-SPM state. However, the two absolute values are larger than the corresponding ones, calculated from the above model: $M([110])=4.67 \mu_{B}$ and $M([120])=4.00 \mu_{B}$, but the extrapolation of the high-field plateaus down to $H=0$ (solid lines in Fig.18) yields magnetizations very close to these calculated values. As in the case of TbPtIn, the slight increase of the magnetization plateaus after the supposed saturation may be caused by the slow approach of the CEF splitting energy.

In calculating the above expected magnetization values, we assumed the easy axes to be along the [110]equivalent directions, based on the directions where maximum magnetization values at $H=70 \mathrm{kG}$ were achieved (fig.18). This is consistent with the angular dependent magnetization measurement for $H=70 \mathrm{kG}$ shown in Fig.19, where angle $\theta$ was measured from the [110] direction; thus the six-fold symmetric magnetization has maxima occurring for the [110]-equivalent directions (i.e. for $\theta=n * 60^{\circ}$, where $n$ is an integer). Consequently, for TmAgGe the angle $\theta$ will be measured from the closest [110] easy axis. The comparison of TmAgGe and TbPtIn indicates that, even though the easy axes in the two compounds correspond to the two different sets of six-fold symmetry directions, as we shall see, their physical properties are very similar.

Around each easy axis, these magnetization measurements follow the $\cos \theta$ angular dependence (the solid line in Fig.19), as expected within our proposed model. Some differences between the experimental data (filled circles in fig.19) and the calculated magnetization could be caused by small misalignment of the sample (rendering slightly asymmetric measured peaks), or by the strong interactions between the local moments. Similar to TbPtIn, this also indicates that different metamagnetic states cross $H=70 \mathrm{kG}$ at different angles.

From the $\left.M(T)\right|_{H, \theta}$ (fig.20) and $\left.M(H)\right|_{T, \theta}$ (fig.21) measurements, detailed $H-T$ phase diagrams for this compound can be determined. They are shown in Fig.22a,c, for field along the [110] or $\theta=0^{0}$, and [120] or $\theta=30^{\circ}$ directions respectively, with an intermediateposition $\theta \approx 24^{0}$ phase diagram in Fig.22b. In the same manner used for TbPtIn, the points in these phase diagrams have been obtained from maxima in either $d(M * T / H) / d T$ for constant field (full symbols) or in $d M / d H$ for fixed temperatures (open symbols).

For $H \|[110]$ (fig.22a), at low temperatures the antiferromagnetic, AF, ground state exists for $H \leq 3.1 \mathrm{kG}$, followed by a small intermediate phase $M_{1}$ (up to $\sim 4.4$ $\mathrm{kG}$ ) and a larger state $M_{2}$ above. This latest phase extends up to $8.9 \mathrm{kG}$, after which, at low temperatures, the system reaches the crystal field-limited saturated paramagnetic, CL-SPM, state. As temperature is increased, the $M_{1}$ phase disappears around $2.5 \mathrm{~K}$, and a direct transition from the $A F$ to the $M_{2}$ state occurs at a decreasing critical field value. The upper phase boundary (for the $M_{2}$ to the PM state transition) also falls down in field as $\mathrm{T}$ increases, such that at very low fields only one transition is observed close to $T_{N}=4.2 \mathrm{~K}$.

As we rotate away from the easy axis, the low-field phase diagram changes very little, with a small enhancement of the critical field values towards low temperatures. As field is being increased, $M_{2}$ is getting smaller as a new distinct phase $M_{3}$ forms. Its upper bordering line appears to have a strong angular dependence, as can be seen in Fig.22b and c, similar to the upper-most phase boundary seen in TbPtIn : for $H \|[120]$ (Fig.22c), this phase boundary is an almost vertical line at $T \approx 4.5 \mathrm{~K}$, up to our maximum applied field $H=70 \mathrm{kG}$. As a consequence, the corresponding points on this line have been determined from $d(M * T / H) / d T$ data, as shown in Fig.20c for high fields, and could not be identified in the field-dependent derivatives. At low temperatures, the $H_{c A F, 1}$ and $H_{c 1,2}$ values $(3.62 \mathrm{kG}$ and $4.86 \mathrm{kG}$ respectively) are very close to the corresponding ones in the [110] direction, whereas the $M_{2}$ to $M_{3}$ transition occurs around $7.0 \mathrm{kG}$. These three phase lines merge around $T=3.0 \mathrm{~K}$, such that for higher temperatures a single transition occurs at decreasing fields. This line appears to intersect the $H=0$ axis around $T_{N}=4.2 \mathrm{~K}$.

The intermediate-orientation phase diagram presented in Fig.22b allowed us to observe the upper-most phase line moving down in field at low temperatures, such that for $\theta \approx 24^{0}$, it intersects the $T=0$ axis close to $40.1 \mathrm{kG}$. In this orientation, this phase boundary can be identified in the $\mathrm{M}(\mathrm{H})$ derivative, as shown in Fig.23 for $T=1.85 \mathrm{~K}$. However, the high field peak in $d M / d H$ is poorly defined, making the determination of the corresponding critical field value more difficult. For a more precise estimate, another criterium was used together with the derivative maxima, as illustrated in the inset in Fig.23: the mid-point (large dot) on the highest-slope linear fit (solid line) of the magnetization data around the transition. Also shown is the error bar for this critical field value, as determined from the two criteria used here.

The fact that this line is now apparent in both $M(T)$ and $M(H)$ data is further confirmation that this phase boundary exists, whereas at lower fields, the only noticeable difference from the $H \|[120]$ direction is the persistence of the $M_{2}$ state up to higher (i.e. $\sim 3.5 \mathrm{~K}$ ) temperatures.

A number of similarities between TbPtIn and TmAgGe have already been established: same crystal structure, antiferromagnetic ground state, extremely anisotropic magnetization, in-plane anisotropy and metamagnetism leading to crystal field-limited saturated magnetizations smaller than the calculated single ion $\mu_{\text {sat }}$ values. As a consequence, we proceed to study the angular dependence of the planar metamagnetism in TmAgGe, and subsequently apply the model developed for TbPtIn to the case of this compound.

When we fix the temperature at $T=2 \mathrm{~K}$, the angular dependence of the metamagnetic transitions can be 
studied based on the $M(H)$ isotherms shown in Fig.24. The critical fields and the locally saturated magnetization values (full symbols in fig.25) have been determined as maxima in $d M / d H$, and from on-set values respectively (see the TbPtIn section). An exception was made for $M_{C L-S P M}$ above $10^{\circ}$, and the criterion used for determining this state is described below. Moreover, because of the proximity of the first two transitions, the $M_{1}$ state is poorly defined; no precise saturated magnetization data could be extracted for this state, but the phase diagrams in Fig.22, as well as the angular dependent critical fields in Fig.25a, are consistent with the existence of this phase. As before, the open symbols in Fig.25 represent reflections of the measured data across the $\theta=0^{0}$ direction. The TmAgGe measurements allowed us to determine the critical field and locally saturated magnetization values for the full angular range $\left(-30^{0} \leq \theta \leq 30^{0}\right)$; the resulting somewhat asymmetric data (most obvious in the case of the $M_{2}$ data) maybe due to a small sample misalignment. The experimental data (full symbols) together with the reflections (open symbols) in Fig.25 give the caliper of the error bars for these measurements.

For $\theta \leq 10^{0}$, two closely spaced metamagnetic transitions can be seen in Fig.25a, with critical fields $H_{C A F, 1}=3.0 \mathrm{kG}$ and $H_{c 1,2}=4.37 \mathrm{kG}$ respectively at $\theta=0^{0}$, followed by a third transition $H_{C 2, C L-S P M}$ at $\sim 9.37 \mathrm{kG}$. It should be noted that these $H_{c i, j}$ values are slightly different from the corresponding ones $(3.10 \mathrm{kG}$, $4.36 \mathrm{kG}$ and $8.92 \mathrm{kG}$ respectively at $T=2.0 \mathrm{~K}, \theta=0^{0}$ ) in the $H-T$ phase diagrams (Fig.22a), as they have been determined from two distinct measurements. Thus small errors in the angular position $\left( \pm 1^{0}\right)$ may convert into small errors in the critical field values $(\leq 3 \%)$.

Somewhat larger differences between the two data sets are observed for $\theta \geq 10^{0}$, specifically for $H_{c 3, C L-S P M}$, which varies more rapidly with the angle than any other critical field. In this angular region, the two lower transitions occur at almost the same critical fields as below $10^{0}$, whereas the critical field for the third one slowly decreases with angle, as a fourth transition appears and rapidly moves up in field. Consequently, the $M_{1}$ metamagnetic state changes little with the angle, whereas the $M_{2}$ state narrows down as the bordering critical fields move closer to each other. The fourth transition being very broad makes the determination of the $M_{3}$ state fairly difficult. Also, with $H_{c 3, C L-S P M}$ broadening out and rapidly moving towards our field limit (i.e. $70 \mathrm{kG}$ ), it was difficult to get a meaningful linear fit of the $M(H)$ curves during the $M_{3}$ to $C L-S P M$ transition; instead we used the intersection of the maximum slope line corresponding to the $M_{2}$ to $M_{3}$ transition, and the best linear fit of the highest magnetization state, to determine $M_{C L-S P M}$ for $\theta \geq 10^{0}$ (Fig.24b, inset).

The best fits to the experimentally measured angular dependent data are shown in Fig.25 as dotted lines. We will use these fits to infer the net distribution of moments as multiples $\mathrm{S}$ of three Ising-like systems, similar to the case of TbPtIn. As mentioned before, we infer that the $M_{1}$ state should exist based on the angular dependent critical fields in Fig.25a, and the $T=2.0 \mathrm{~K}$ metamagnetic phases revealed by the phase diagrams in Fig.22. Consequently, in Fig.25b we are only showing the expected angular dependence of such a phase, by analogy with the TbPtIn case: $M_{1}^{\text {calc }} / \mu_{\text {sat }}\left(\mathrm{Tm}^{3+}\right)=2 / 18 * \cos \theta$, which appears to be the upper limit of these magnetization values, as indicated by the error bars shown in Fig.25b. As already seen for TbPtIn, the moment configuration that would result in such a $M_{1}$ magnetization is a $S=6$ state: $(\searrow \searrow \searrow \searrow \searrow \nwarrow \uparrow \uparrow \uparrow \uparrow \uparrow \uparrow \swarrow \swarrow \swarrow \swarrow \swarrow \nearrow)$ or $(\searrow \searrow \searrow \nwarrow \nwarrow \downarrow \downarrow \uparrow \uparrow \uparrow \uparrow \swarrow \swarrow \swarrow \nearrow \nearrow \nearrow) . M_{2}(\theta)$ has a maximum value at $\theta=0^{0}$ equal to $2.37 \mu_{B} / \mathrm{Tm}^{3+}$, close to $2 / 6 * \mu_{\text {sat }}\left(\mathrm{Tm}^{3+}\right)=2 / 6 * 7 \mu_{B} / \mathrm{Tm}^{3+}$, and a $\cos \theta$ angular dependence. This suggests that a possible net distribution of moments for this state, realized with a minimum $S=2$, could be $(\searrow \nwarrow \uparrow \uparrow \swarrow \nearrow)$. If one local moment is flipped from its $\measuredangle$ position to $\nearrow$ in the previous state, the resulting state could be described by the $(\searrow \nwarrow \uparrow \uparrow \nearrow \nearrow)$ configuration, whose magnetization varies as $2 \sqrt{3} / 6 * \mu_{\text {sat }}\left(\mathrm{Tm}^{3+}\right) * \cos \left(\theta-30^{0}\right)$; this fits well the measured $M_{3}$ data, indicating that the previously assumed local moment distribution may be appropriate for this metamagnetic state. When all the magnetic moments are in their 'up' positions, the CL-SPM $(\nwarrow \nwarrow \uparrow \uparrow \nearrow \nearrow)$ state is achieved, and the corresponding angular dependence is $4 / 6 * \mu_{\text {sat }}\left(\mathrm{Tm}^{3+}\right) * \cos \theta$. This best describes the last observed metamagnetic state, which has a maximum of $4.68 \mu_{B} / \mathrm{Tm}^{3+}$ around $\theta=0^{0}$, very close to $4 / 6 * \mu_{\text {sat }}\left(\mathrm{Tm}^{3+}\right)=4 / 6 * 7 \mu_{B} / \mathrm{Tm}^{3+}$. (As before, the order of the arrows used to describe the net distribution of moments has no physical meaning).

According to the calculation given in the case of TbPtIn, for TmAgGe one would also expect the critical fields to vary with the angle $\theta$ as

$$
H_{c i, j} \sim \frac{1}{M_{j}-M_{i}} .
$$

Using the net distributions of moments assumed above to best describe the locally saturated magnetization states, we expect the following angular dependencies of the critical fields:

$$
\begin{aligned}
& H_{C A F, 1}(\theta) \sim 1 / \cos \theta, \\
& H_{c 1,2}(\theta) \sim 1 / \cos \theta \\
& H_{c 2,3}(\theta) \sim 1 / \cos \left(\theta-60^{0}\right), \\
& H_{c 3, C L-S P M} \sim 1 / \cos \left(\theta+60^{\circ}\right) \\
& \text { and } H_{C 2, C L-S P M}(\theta) \sim 1 / \cos \theta .
\end{aligned}
$$

The experimental data (full symbols in Fig.25a) are in good agreement with these calculated critical fields, with $H_{c 2, C L-S P M}(\theta)$ present only for $\theta \leq 10^{0}$, while the $H_{c 2,3}$ and $H_{C 3, C L-S P M}$ exist only for $\theta \geq 10^{\circ}$. This is consistent with the presence of the $M_{3}$ state for angles larger than $10^{\circ}$, even though experimentally we were only able to accurately determine it for $\theta \geq 16^{0}$. (The bordering transitions of this state are very close in field when $\theta$ is close to $10^{\circ}$, which made the determination of $M_{3}$ in this angular region difficult). It is worth pointing out the excellent fit of the measured $H_{c 3, C L-S P M}$ data with the calculated angular dependency, this transition 
showing the most dramatic change with the angle $\theta$.

Apart from the absolute values of the critical fields and the locally saturated magnetizations, the $H_{c}(\theta)$ and $M_{\text {sat }}(\theta)$ phase diagrams for TmAgGe (Fig.25) are identical to the TbPtIn analogues in Fig.13: to the same number of critical fields with identical angular dependencies correspond identical metamagnetic values (scaled to the saturated moment of the respective $\mathrm{R}^{3+}$ ion), which also vary similarly with the angle. This is consistent with our model being indeed a general description of the $\mathrm{Fe}_{2} \mathrm{P}$ type systems, or even more generally, of hexagonal systems with the $\mathrm{R}$ in orthorhombic point symmetry.

When the $H_{c}(\theta)$ phase diagram for TmAgGe is converted into a polar plot (Fig.26), similar to that for TbPtIn, we again notice that the phase boundaries are straight lines, with $\pm 1 / \sqrt{3}$ or $\infty$ slopes. From the equations of these straight lines, we can once more confirm the transitions determined before:

$$
\begin{aligned}
& H_{c}(\theta) \sim 1 / \cos \theta \text { for } b=\infty \\
& H_{c}(\theta) \sim 1 / \cos \left(\theta-60^{\circ}\right) \text { for } b=-1 / \sqrt{3} \\
& \text { or } \\
& H_{c}(\theta) \sim 1 / \cos \left(\theta+60^{\circ}\right) \text { for } b=1 / \sqrt{3} .
\end{aligned}
$$

As already noted, in the case of TmAgGe, we were able to determine the critical fields from experimental data for $\theta=-30^{\circ} . .30^{\circ}$, as seen in the phase diagrams in Fig.25a, as well as in Fig.26. Slight differences between the expected straight lines in Fig. 26 and measured critical fields can be noticed for $H_{c 2,3}$ for angles close to $\pm 30^{\circ}$, or for $H_{c 3, C L-S P M}$ also for large angles; besides being a consequence of small misorientation of the sample, this may indicate, similar to the TbPtIn case, that a more complex model needs to be used to describe the regions around the 'hard' in-plane direction.

\section{ANGULAR DEPENDENT METAMAGNETISM AT $T=20$ K IN TbPtIn}

Whereas TmAgGe has fairly simple $H-T$ phase diagrams, with all metamagnetic phases present at low temperatures, the TbPtIn phase diagrams are somewhat more complex, manifesting an additional intermediatetemperature phase, $M_{4}$ (Fig.9). In order to perform a similar angular dependent study of this metamagnetic state, a set of $\left.M(H)\right|_{\theta}$ data was taken at $T=20 \mathrm{~K}$. Assuming that all existing transitions have been identified and are shown in fig.9 (at least below $20 \mathrm{~K}$ ), at this temperature the $M(H)$ curves should intersect the same magnetic phases as in the low temperature case, with the exception of $M_{1}$; instead, the measurements at $T=20$ $\mathrm{K}$ intersect the bubble-like phase $M_{4}$, as seen in the three different orientations phase diagrams in Fig.9.

Fig. 27 shows the $M(H)$ isotherms at $T=20 \mathrm{~K}$ for various angles $\theta$. The $M_{j}(\theta)$ and $H_{c i, j}(\theta)$ phase diagrams have been determined as described before for TbPtIn or TmAgGe for $T=2 \mathrm{~K}$, and are shown in fig.28. It should be noted that, due to the enhanced temperature, all transitions are broadened, and the locally saturated magnetization plateaus are no longer horizontal. Both of these facts make the analysis of these data somewhat harder and more ambiguous.

For $\theta \leq 12^{0}$ (Fig.28a), the lowest transition changes very little with angle, having a critical field value $H_{c A F, 4} \approx 8 \mathrm{kG}$. As field is being increased, two more transitions can be observed for angles lower than $8^{0}$, with local minima of the critical fields, at $\theta=0^{0}$, of $H_{c 4,2}=16.2 \mathrm{kG}$ and $H_{c 2, C L-S P M}=48.7 \mathrm{kG}$ respectively. For larger angles, the highest transition splits into two different ones, $H_{c 2,3}$, with decreasing values as we rotate away from the easy axis, and $H_{c 3, C L-S P M}$, which rapidly increases above our field limit (i.e. $55 \mathrm{kG}$ ) around $\theta=12^{0}$. It should be noted that we are still referring to the high-field state at $T=20 \mathrm{~K}$ as the crystal field-limited saturated paramagnetic CL-SPM state, even though it is possible that cross-over to the paramagnetic PM state has occurred between $2 \mathrm{~K}$ and $20 \mathrm{~K}$ at high $H$. (This would be a plausible explanation for the measured magnetization values for this high field state being, as seen below, lower than the calculated values.)

The locally saturated magnetization of the $M_{4}$ state is equal to $\sim 0.25 \mu_{B} / \mathrm{Tb}$ at $\theta=0^{0}$ and doesn't appear to change much with the angle. According to the $H-T$ phase diagrams in Fig.9, all higher metamagnetic states are identical at low $(T=2 \mathrm{~K})$ temperature and at $T=20 \mathrm{~K}$; consequently, they seem to have similar angular dependencies (fig.28b): $M_{2}$ and $M_{C L-S P M}$ have local maxima around $3.3 \mu_{B} / \mathrm{Tb}$ and $5.7 \mu_{B} / \mathrm{Tb}$ respectively, at $\theta=0^{0}$, and slowly decrease with increasing angle in this region. Beyond $\sim 8^{0}$, a third metamagnetic state should exist, defined by the $H_{c 2,3}$ and $H_{c 3, C L-S P M}$ critical fields; however, it is difficult to identify it in this angular region, given the broadness of the bordering transitions and the $H_{c 3, C L-S P M}$ proximity to our field limit.

As we move further away from the easy axis (i.e. $\theta>12^{0}$, Fig.28b), the lower two metamagnetic states can again be observed, whereas the CL-SPM state may still exist for fields larger than $55 \mathrm{kG}$ (also apparent from the $H-T$ phase diagram in Fig.9b). Also, we can now see the third state $M_{3}$ slowly increasing with angle $\theta$, similar to the low temperature case.

The $T=20 \mathrm{~K}$ phase diagrams (fig.28) are very similar to their low temperature analogues (Fig.13), except for the $M_{4}$ state, and some evident differences between the experimental data and the model calculations (dotted lines). It was rather difficult to determine the angular dependencies of $M_{4}$ and $M_{2}$, therefore we had to infer the possible theoretical fits in a more indirect way: as we already mentioned, the state described by $M_{2}$ at $T=20 \mathrm{~K}$ should be the same as the corresponding one at low temperature, since they characterize the same metamagnetic phase. Therefore we expect it to vary with the angle like $2 / 6 * \mu_{\text {sat }}\left(\mathrm{Tb}^{3+}\right) * \cos \theta$ (fig.13b). For large angles, this is consistent with the measured data in Fig.28b, whereas significant deviations can be noticed closer to $\theta=0^{0}$. The lower magnetization state $M_{4}$ has much smaller values than any of the states characterized at $T=2 \mathrm{~K}$, 
therefore we cannot fit it accurately with a calculated angular dependence; however, $H_{c 4,2}$ can be fitted with 16.2 $\mathrm{kG} * 1 / \cos \theta$ and should relate to $M_{4}$ through

$$
H_{c 4,2}(\theta) \sim 1 /\left[M_{2}(\theta)-M_{4}(\theta)\right]
$$

or

$1 / \cos \theta \sim 1 /\left[2 / 6 * \mu_{\text {sat }}\left(\mathrm{Tb}^{3+}\right) * \cos \theta-M_{4}(\theta)\right]$.

Thus $M_{4}(\theta)$ should vary like $M_{s a t, 4} * \cos \theta$, with a locally saturated magnetization $M_{\text {sat }, 4} \approx 0.25 * \mu_{\text {sat }}\left(\mathrm{Tb}^{3+}\right)$, but, as already mentioned, it is difficult to determine it with reasonable accuracy. However, the corresponding local moment configuration should be similar to the low temperature one, in order to get the $\cos \theta$ dependence, except that the number $\mathrm{S}$ of three Ising-like systems which would yield the appropriate $M_{\text {sat,4 }}$ value is uncertain.

All other locally saturated magnetizations and critical fields can be best fitted with the same angular dependencies as for the low temperature case:

$$
\begin{aligned}
& M_{3}(\theta)=2 \sqrt{3} / 6 * \mu_{\text {sat }}\left(\mathrm{Tb}^{3+}\right) * \cos \left(\theta-30^{0}\right), \\
& M_{C L-S P M}(\theta)=4 / 6 * \mu_{\text {sat }}\left(\mathrm{Tb}^{3+}\right) * \cos \theta, \\
& \text { and } \\
& H_{c A F, 4} \sim 1 / \cos \theta, \\
& H_{c 2,3} \sim 1 / \cos \left(\theta-60^{0}\right), \\
& H_{c 2, C L-S P M} \sim 1 / \cos \theta, \\
& H_{c 3, C L-S P M} \sim 1 / \cos \left(\theta+60^{0}\right) .
\end{aligned}
$$

Apart from the already mentioned differences between the measured data and the calculated curves, small departures from the corresponding theoretical angular dependencies can be noticed for $M_{3}(\theta)$ and $H_{c 2,3}$; a more significant difference appears for the saturated magnetization state $M_{C L-S P M}(\theta)$, which seems to have the expected angular dependence, but with smaller values than the calculated ones. This may be a high-temperature effect (i.e. cross-over from low-T CL-SPM state to high-T paramagnetic PM state), or it may be one more indication that a more refined model is needed.

A polar plot analogues to the low temperature case (Fig.29) shows that at $T=20 \mathrm{~K}$, the critical fields are still well described by straight lines, but with more pronounced differences between experiment and the theoretical calculations. We attribute these to the thermal broadening at this temperature, but, as already seen in the low temperature case, a requirement for a more complex model cannot be excluded.

\section{SUMMARY}

Motivated by the extensive work done on highly anisotropic local moment systems with tetragonal unit cells and unique rare earth sites of tetragonal point symmetry, we have performed detailed studies on two highly anisotropic, local moment, hexagonal compounds: TbPtIn and TmAgGe. Whereas both of these compounds are ternary members of the $\mathrm{Fe}_{2} \mathrm{P}$ class of materials, they have different ligands. In addition, whereas both of these compounds manifest extreme planar anisotropy, they have different easy axes: [120] for TbPtIn and [110] for TmAgGe. Even with these differences we have found that these two compounds have very similar $H-T$ as well as $H-\theta$ phase diagrams.

TbPtIn and TmAgGe have a single rare earth site with orthorhombic point symmetry (with three rare earth sites per unit cell) and both compounds have high field saturated moments well below the single ion values. These two observations, combined with our experience with the four position clock model that was developed for the tetragonal compounds with rare earths in tetragonal symmetry, lead us to propose a similar model for these $\mathrm{Fe}_{2}$ P-type compounds: a triple coplanar Ising model, which consists of three Ising-like moments per unit cell, with their Ising axes within the basal plane and rotated by $60^{\circ}$ with respect to each other. This model preserves the six-fold symmetry at high fields and also explains why the saturated moments are significantly lower than the free ion values. By analyzing the magnitudes and angular dependencies of the critical metamagnetic fields, as well as the locally saturated magnetizations within the framework of this model, we can infer the net distribution of moments along the six possible moment orientations. However, field-dependent neutron diffraction or magnetic x-ray measurements are needed to test these hypothetical net distributions of moments and to obtain the propagation vectors for each magnetically ordered state. (Some preliminary experiments on TbPtIn are already reported ${ }^{12}$, confirming the two low-field ordering temperatures that we observed in the magnetization measurements. Moreover, the neutron data are consistent with our inferred direction of the moments.)

The successful extension of the four state clock model to the current triple coplanar Ising-like model implies that a wider set of local moment compounds with planar anisotropy can be understood in a similarly simple manner. Clearly tetragonal unit cell compounds with the rare earth in orthorhombic point symmetry could be expected to behave in a manner similar to TbPtIn and TmAgGe, i.e. to form a class of double coplanar Ising model materials. In a similar manner, hexagonal unit cell compounds with the rare earth in hexagonal point symmetry could be expected to behave in a manner similar to $\mathrm{HoNi}_{2} \mathrm{~B}_{2} \mathrm{C}^{3}$ or $\mathrm{DyAgSb}_{2}{ }^{\frac{5}{3}}$, i.e. to form a class of six position clock model materials. Hexagonal unit cell materials may offer one other, potentially new class of materials: highly planar anisotropic compounds with the rare earth in trigonal point symmetry. In this case we anticipate a double coplanar three position clock model. Such compounds would have two magnetic sites, each with three possible positions $120^{\circ}$ apart. Fig.30 gives schematic representations of all expected five models described above.

\section{ACKNOWLEDGMENTS}

We thank Lizhi Tan for helping index the macroscopic facets of our crystals. We are also grateful for fruitful discussions with Carsten Detlefs and Andreas Kreyssig. Ames Laboratory is operated for the U.S. Department of 
Energy by Iowa State University under Contract No. W7405-Eng.-82. This work was supported by the Director for Energy Research, Office of Basic Energy Sciences.

1 S.L. Bud'ko, Z. Islam, T.A. Wiener, I.R. Fisher, A.H. Lacerda and P.C. Canfield, J. Magn. Magn. Matter. 20553 (1999)

2 B.K. Cho, P.C. Canfield and D.C. Johnston, Phys. Rev. B 538499 (1996)

3 P.C. Canfield, S.L. Bud'ko, B.K. Cho, A.H. Lacerda, D. Farrell, E. Johnston-Halperin, V.A. Kalatsky and V.L. Pokrovsky, Phys. Rev. B 55970 (1997)

4 P.C. Canfield and S.L. Bud'ko, J. Alloys Comp. 262-263 169 (1997)

${ }^{5}$ K.D. Myers, P.C. Canfield, V.A. Kalatsky and V.L. Pokrovsky, Phys. Rev. B 591121 (1999)

6 V.A. Kalatsky and V.L. Pokrovsky, Phys. Rev. B 575485 (1998)

7 E. Morosan, S.L. Bud'ko, P.C. Canfield, M.S. Torikachvili and A.H. Lacerda, J. Magn. Magn. Matter. 277298 (2004)

8 Y.V. Galadzhun, V.I. Zaremba, H. Piotrowski, P. Mayer, R.D. Hoffmann and R. Pöttgen, Z. Naturforsch 55b 1025 (2000)

9 K.C. Watson, J. Crangle, K.-U. Neumann and K.R.A. Ziebeck, J. Magn. Magn. Matter. 140-144 883 (1995)

10 M. E. Fisher, Phil. Mag., 71731 (1962)

11 H. Suzuki, D. Gignoux, D. Schmitt, T. Shigheoka, P.C. Canfield and C. Detlefs, J. Magn. Magn. Matter. 25116 (2002)

12 O. Garlea, E. Morosan, S.L. Bud'ko, J.L. Zarestky, P.C. Canfield and C. Stassis, J. Appl. Phys 956921 (2004 ) 


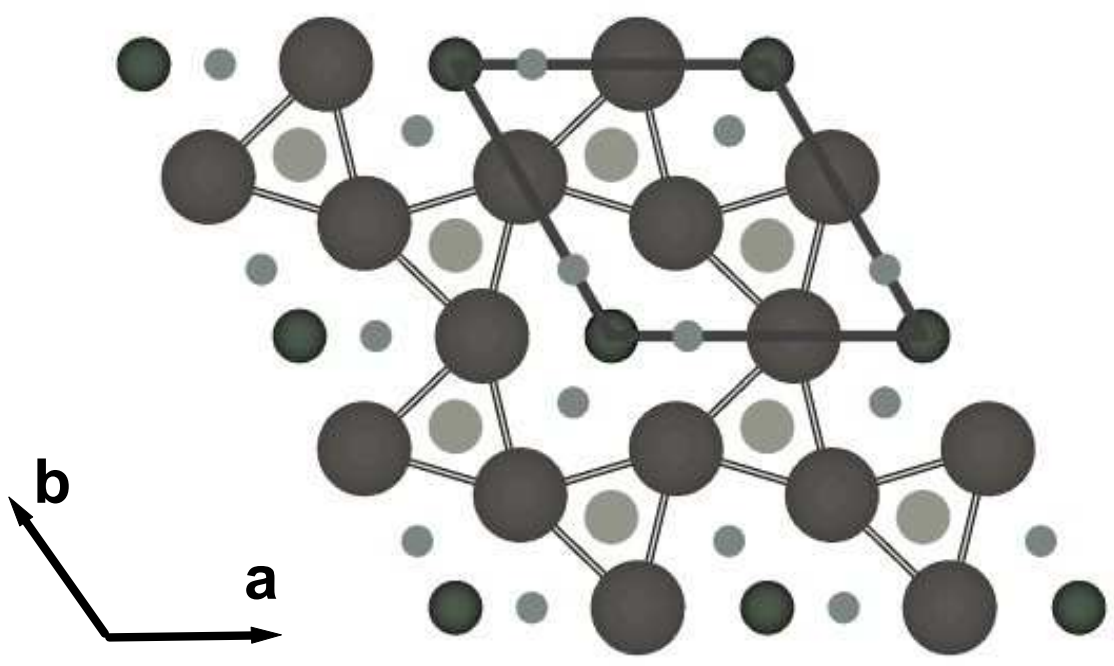

FIG. 1: Projection of the $Z r N i A l$-type crystal structure along the hexagonal c-axis: $\mathrm{R}$-large circles, $\mathrm{M}$ ( $\mathrm{Pt}$ or Ag)-medium circles, $\mathrm{X}$ (In or Ge)- small circles. Light circles: $z=0$ plane, dark circles: $z=1 / 2$ plane. (Note: the $\mathrm{Pt}$ or $\mathrm{Ag}$ atoms (medium circles) appear in both planes.) 


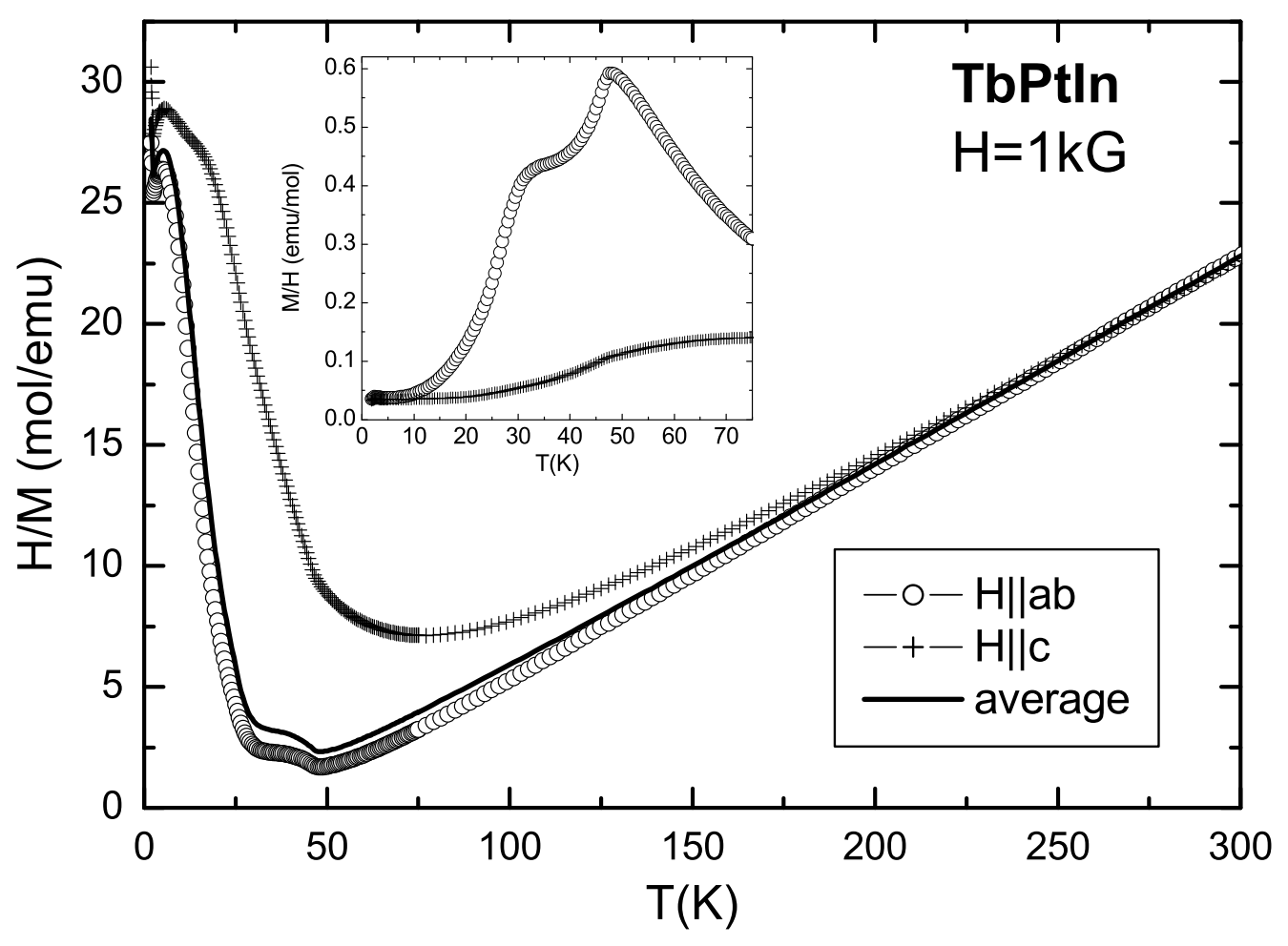

FIG. 2: Anisotropic inverse susceptibility of TbPtIn (symbols) and the calculated average (line); inset: lowtemperature anisotropic susceptibilities. 


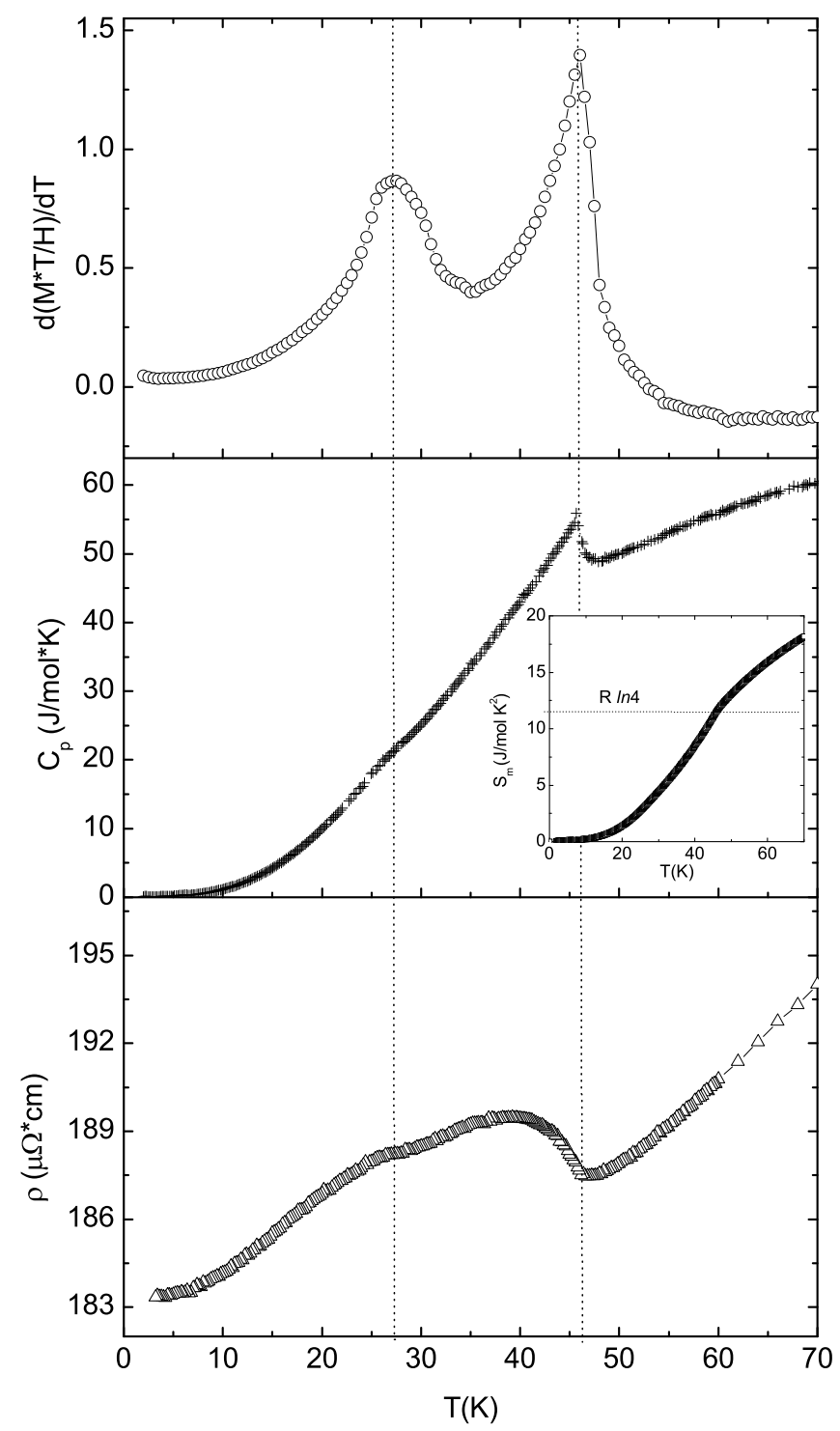

FIG. 3: (a) Low-temperature $d\left(\chi_{a v e} * T\right) / d T$, with vertical dotted lines marking the peaks positions; (b) specific heat $C_{p}(T)$; inset: magnetic entropy $S_{m}(T)$; (c) low-temperature resistivity $\rho(T)$, for current flowing in the basal plane $(i \| a b)$. 


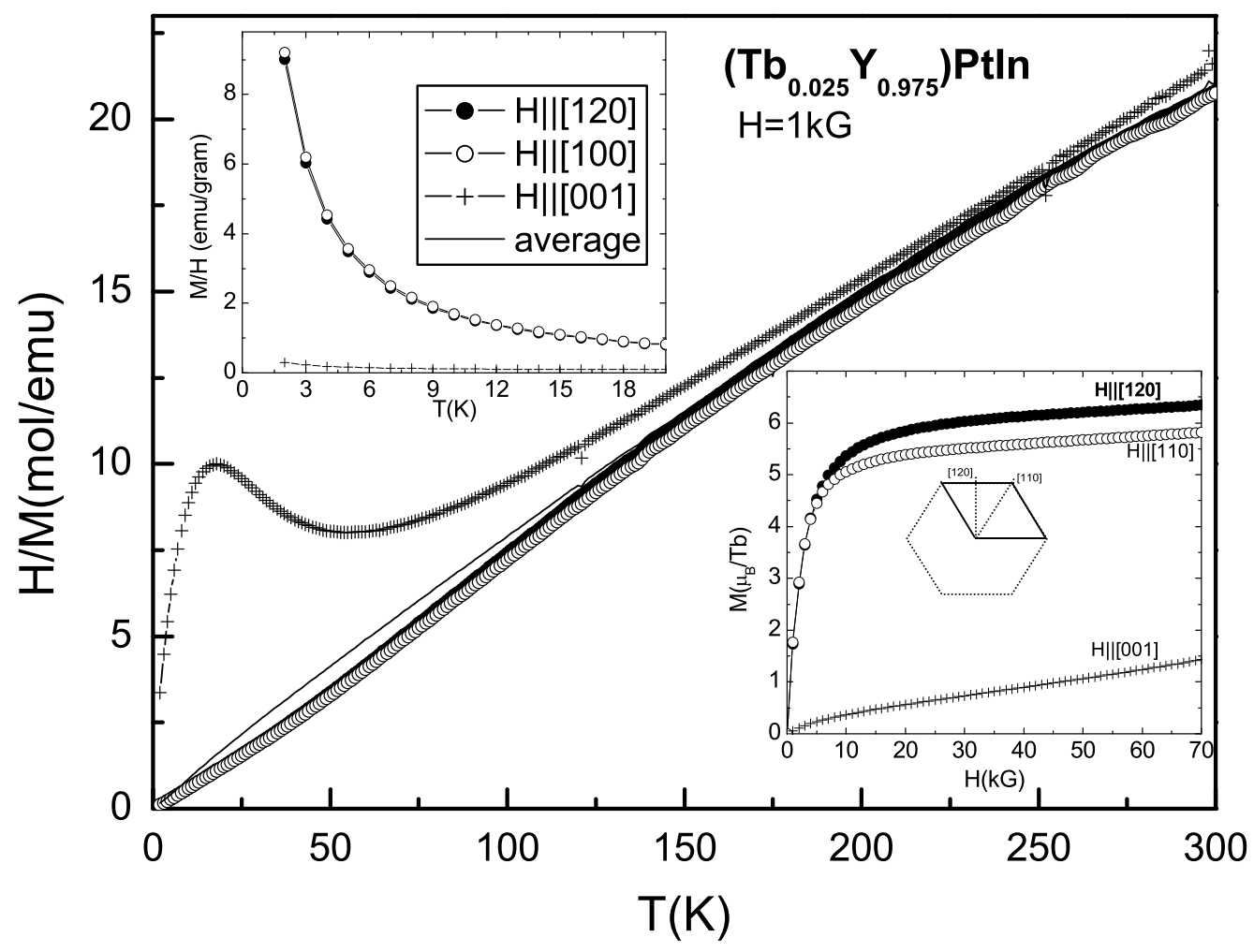

FIG. 4: Anisotropic inverse susceptibility (symbols) and calculated average (line) for $\mathrm{H}=1 \mathrm{kG}$; upper inset: low- temperature anisotropic susceptibility; lower inset: field-dependent anisotropic magnetization, for $\mathrm{T}=2 \mathrm{~K}$. 


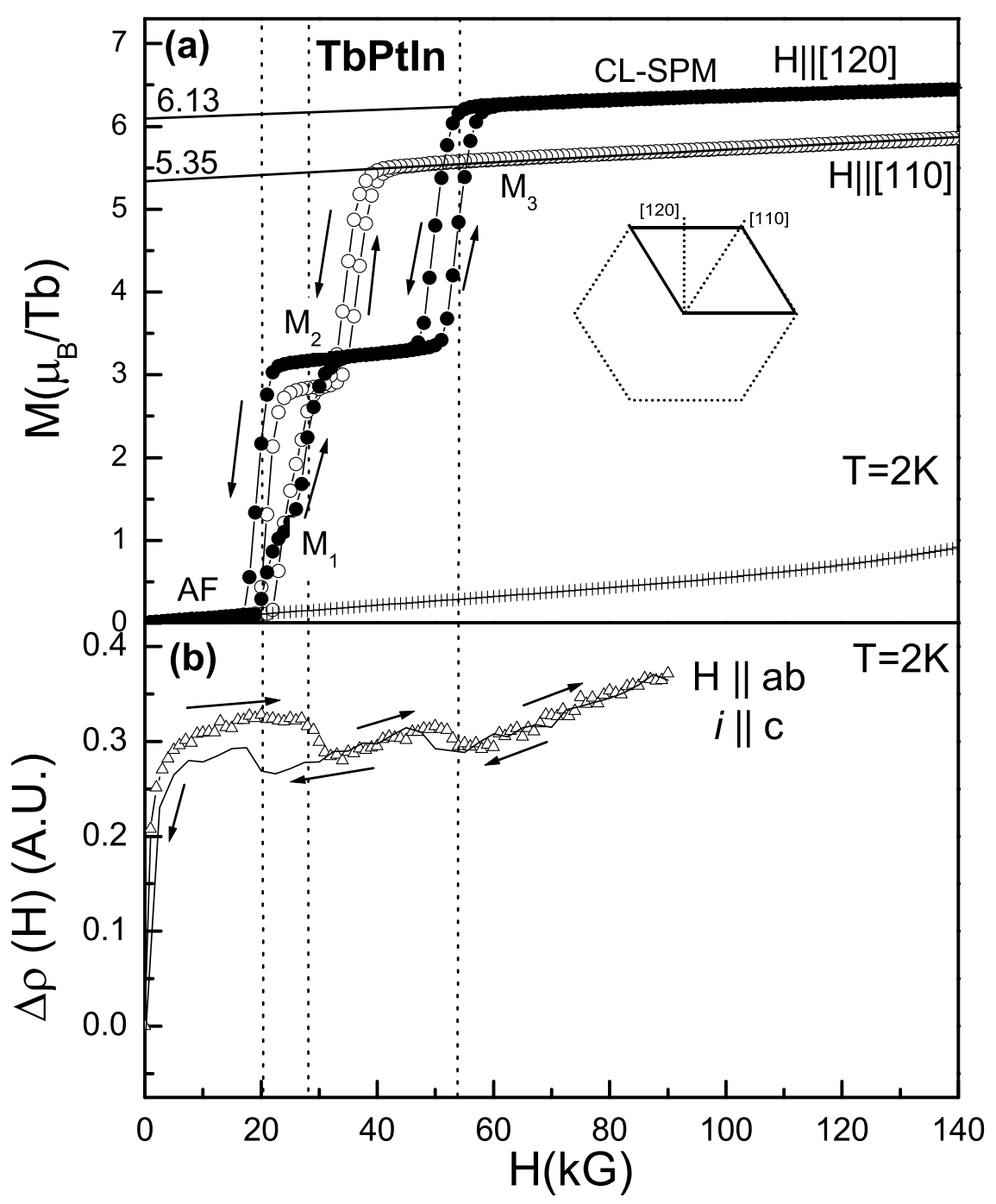

FIG. 5: (a) Anisotropic field-dependent magnetization (increasing and decreasing field data indicated by arrows) and (b) transverse magnetoresistance, for increasing (symbols) and decreasing (line) field. 


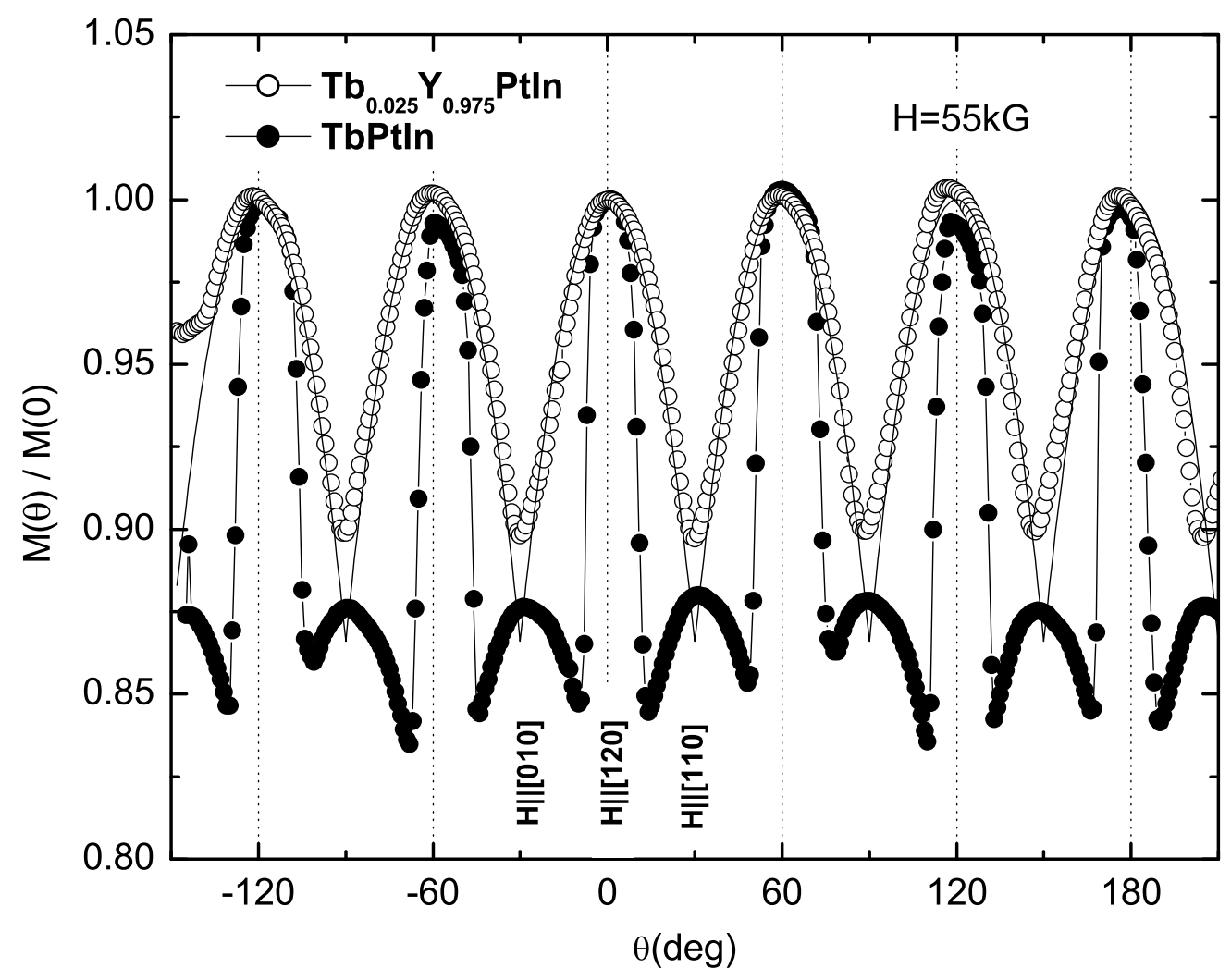

FIG. 6: $\mathrm{M}(\theta)$ of TbPtIn (full circles) and $\mathrm{Tb}_{0.025} \mathrm{Y}_{0.975} \mathrm{PtIn}$ (open circles) for $\mathrm{T}=2 \mathrm{~K}$ and $\mathrm{H}=55 \mathrm{kG}, \mathrm{H} \perp \mathrm{c}$. Solid line represents the calculated $\mathrm{M}_{\max } * \cos \left(\theta-n * 60^{\circ}\right)$, $n$-integer. Note: $\theta=0^{0}$ is defined at the [120] direction. 

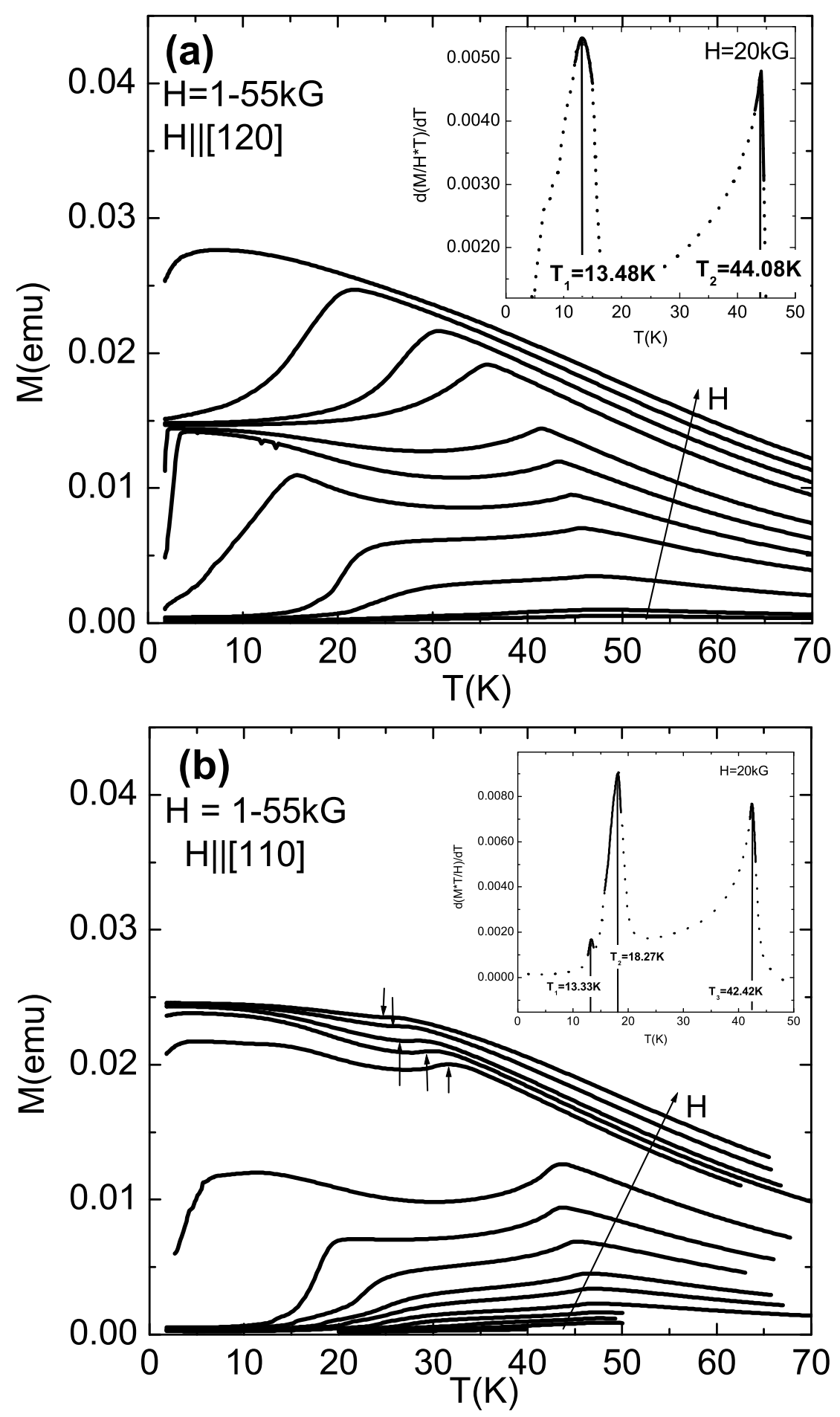

FIG. 7: $\mathrm{M}(\mathrm{T})$ data for various fields for two in-plane orientations of the applied field: (a) $\mathrm{H} \|[120]$ for $\mathrm{H}=1,2,7.5,15$, 20, 25,30,40,45, 50 and $55 \mathrm{kG}$, and (b) $\mathrm{H} \|[110]$ for $\mathrm{H}=$ $1,2.5,3.5,5,7.5,10,15,20,26,30,37.5,40,42.5,45,50$ and $55 \mathrm{kG}$. Insets show enlarged $\mathrm{M}(\mathrm{T}){ }^{*} \mathrm{~T}$ derivatives (dotted lines) for $\mathrm{H}=20 \mathrm{kG}$, together with the Lorentzian fits of the maxima (solid lines), to examplify how the points represented by full symbols on the H-T phase diagrams were determined. 

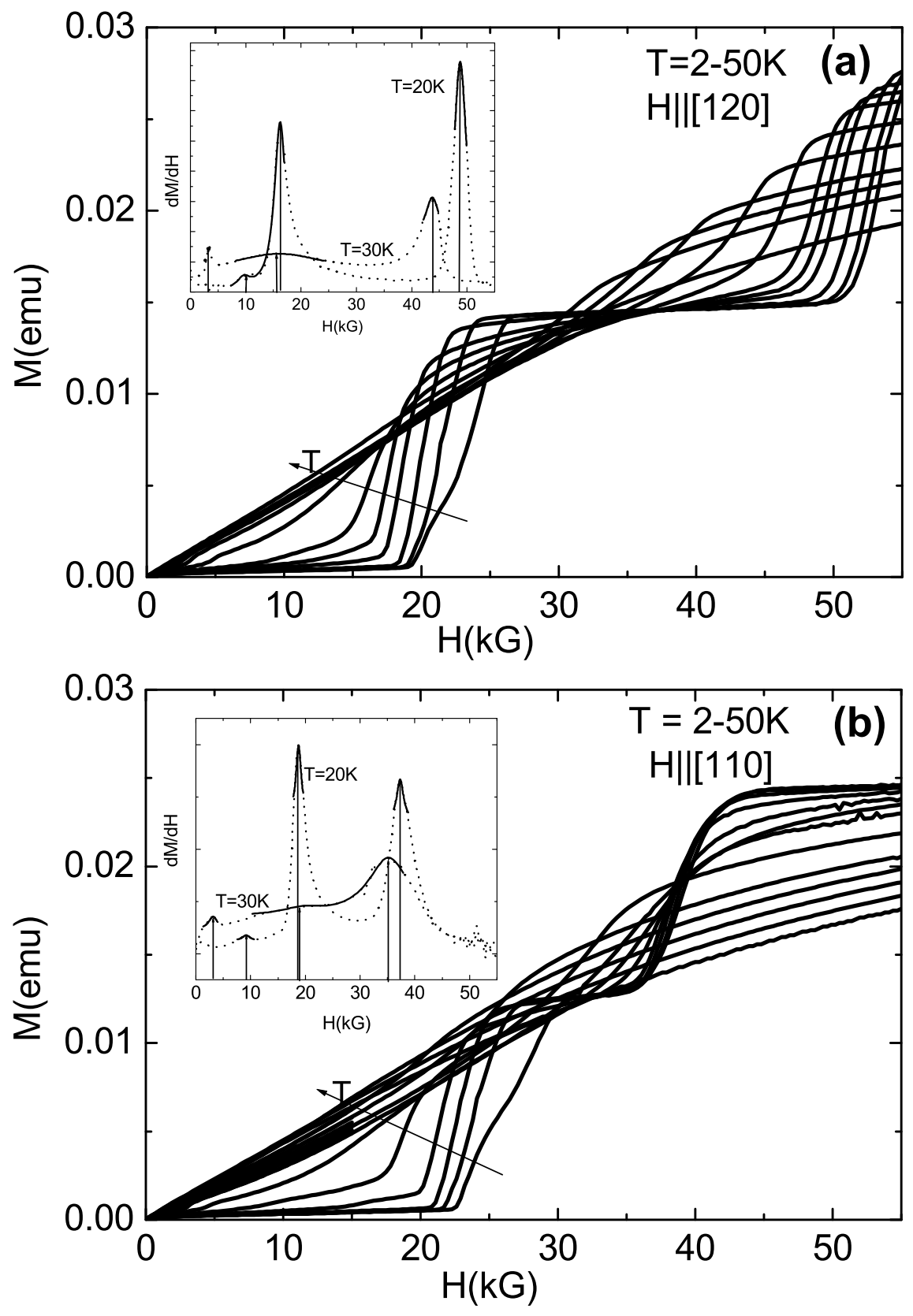

FIG. 8: $\mathrm{M}(\mathrm{H})$ isotherms for two in-plane orientations of the applied field: (a) $\mathrm{H} \|[120]$ for $\mathrm{T}=3,5,10,15,17.5,20,25$, $30,35,37.5,40$ and $45 \mathrm{~K}$, and (b) $\mathrm{H} \|[110]$ for $\mathrm{T}=2,5$, $10,15,20,25,30,35,40,42.5,45$ and $47.5 \mathrm{~K}$. Insets show enlarged $\mathrm{M}(\mathrm{H})$ derivatives (dotted lines) for $\mathrm{T}=20 \mathrm{~K}$ and $30 \mathrm{~K}$, for the corresponding field directions, together with the Lorentzian fits of the maxima (solid lines), to examplify how the points represented by open symbols on the $\mathrm{H}-\mathrm{T}$ phase diagrams were determined. 


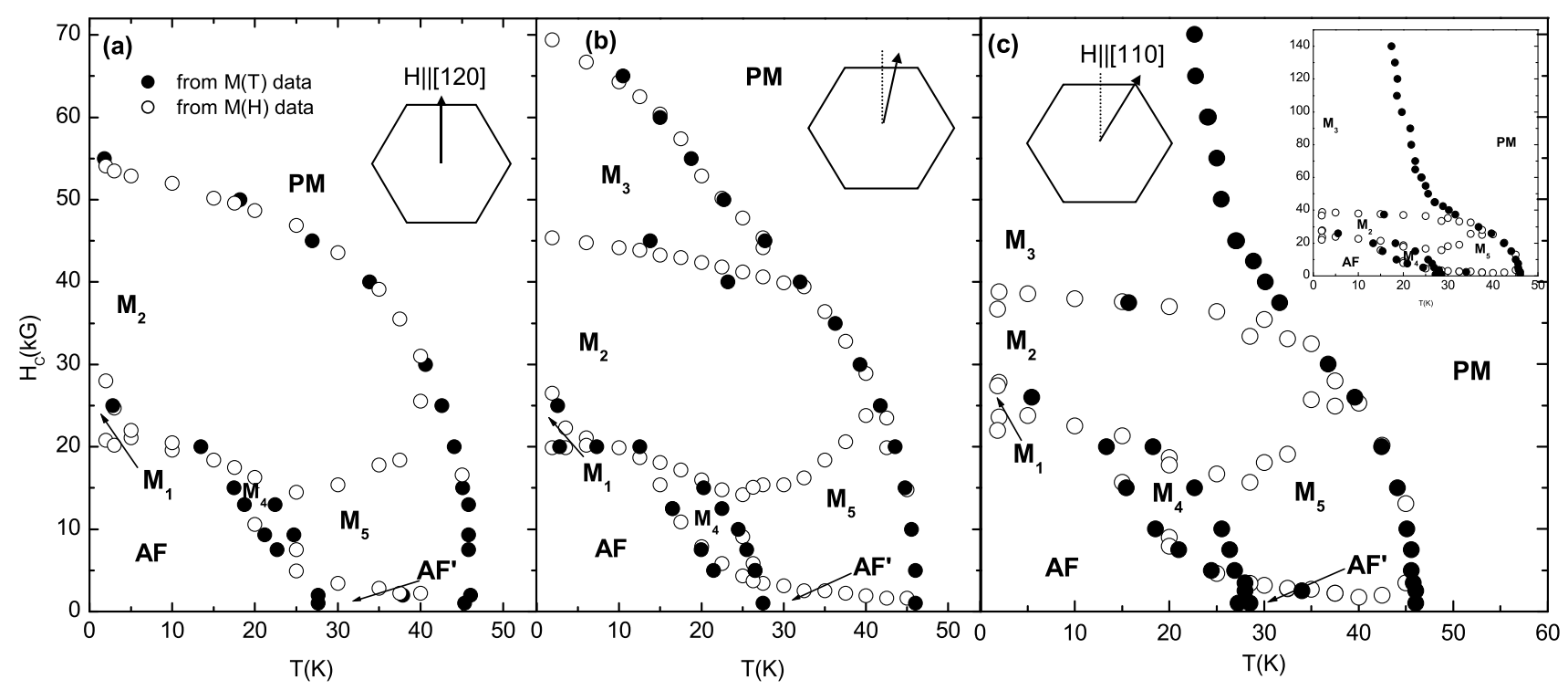

FIG. 9: H-T phase diagrams for (a) H\|[120] and (c) H\|[110], as determined from the magnetization data in Fig.7-8, with an intermediate field orientation phase diagram (see text) shown in (b). 

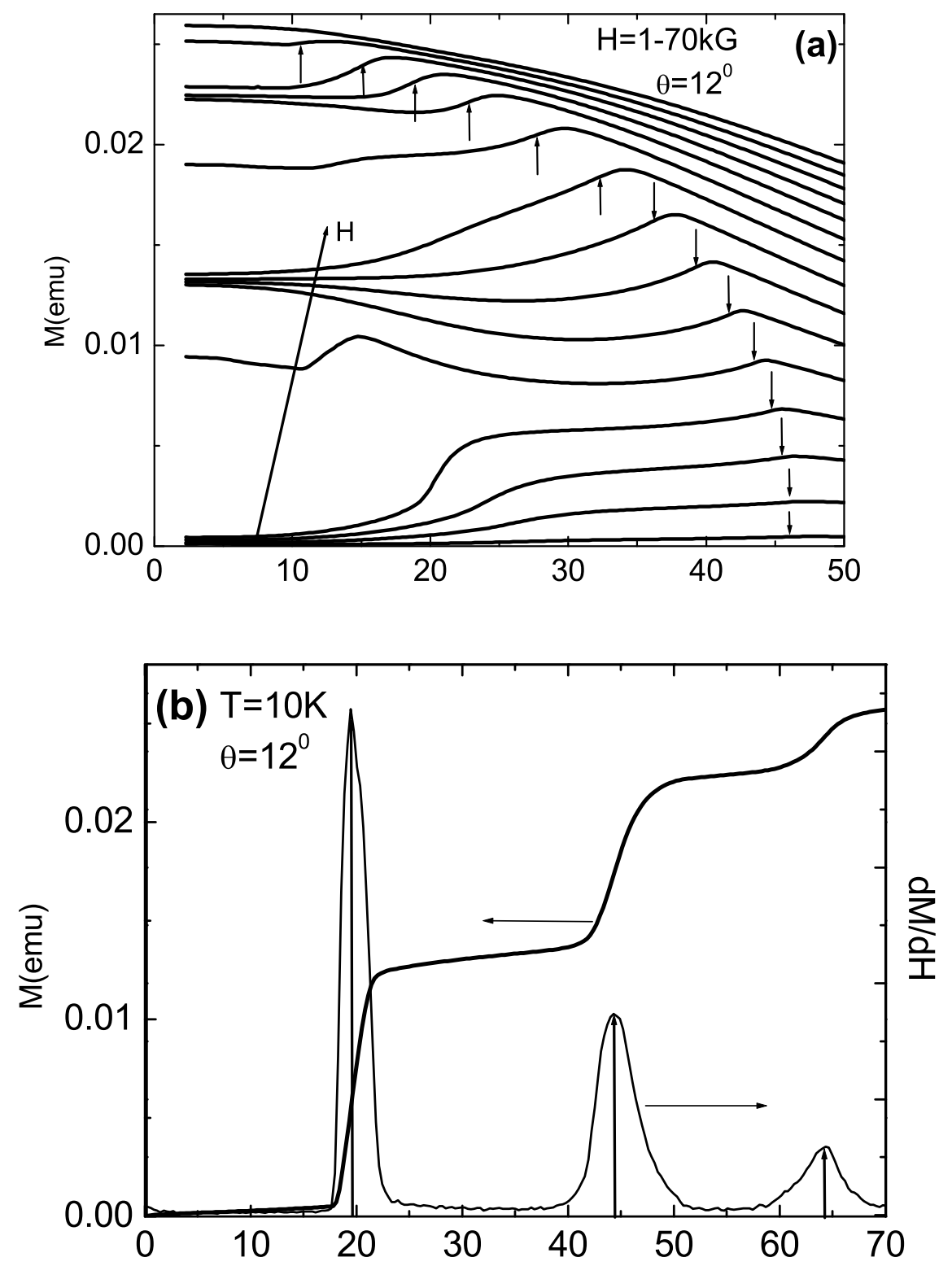

FIG. 10: (a) $\mathrm{M}(\mathrm{T})$ data for various fields: $\mathrm{H}=1 \mathrm{kG}$ and $5-70 \mathrm{kG}(\Delta \mathrm{H}=5 \mathrm{kG})$ for the intermediate field direction (see text). The small arrows indicate the highest-field transitions at the corresponding temperature. (b) $\mathrm{M}(\mathrm{H})$ for $\mathrm{T}=10 \mathrm{~K}$, and the corresponding derivative, as an example of how the open symbols on the upper-most phase line in Fig.9b have been determined. 

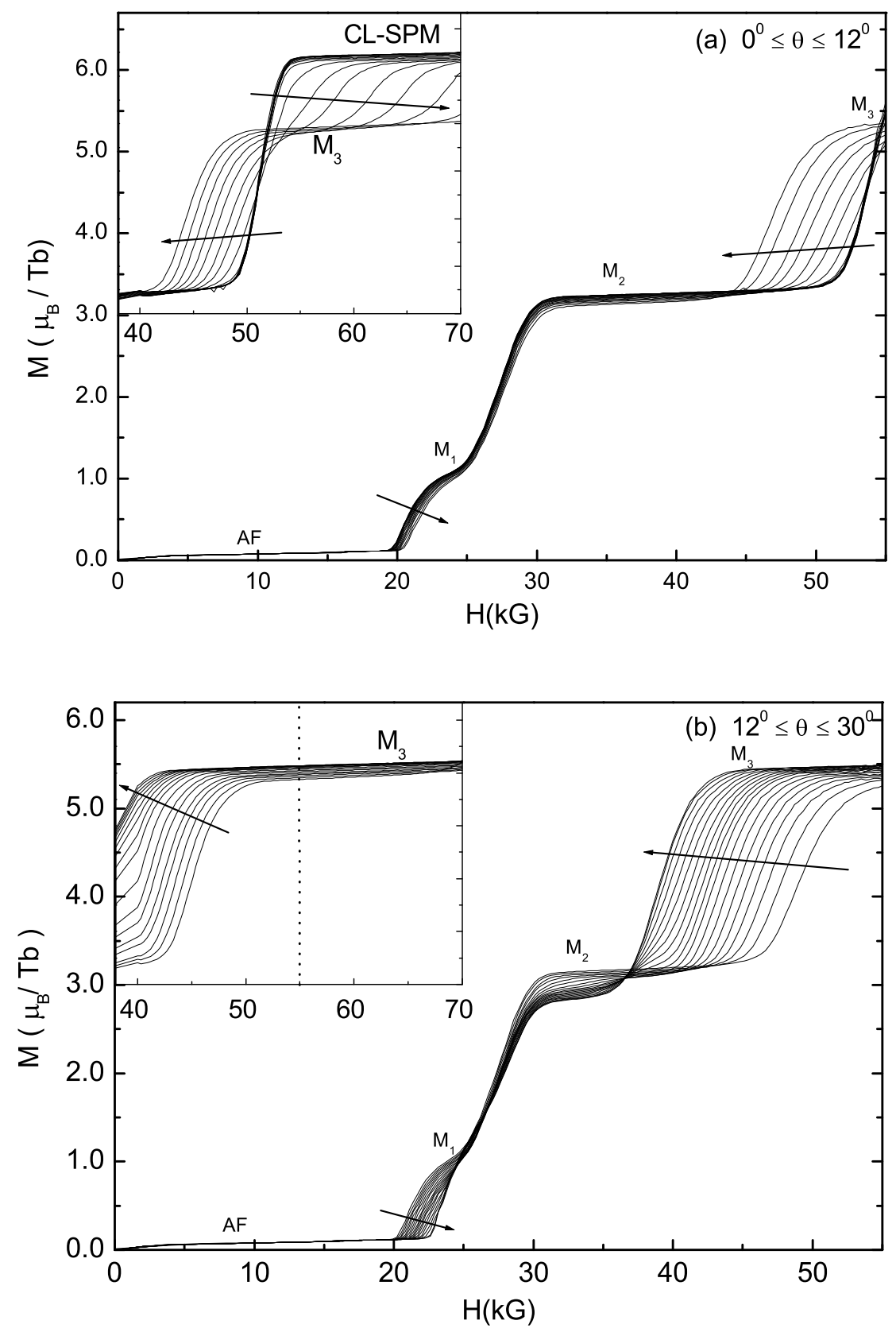

FIG. 11: $\mathrm{M}(\mathrm{H})$ isotherms at $\mathrm{T}=2.0 \mathrm{~K}$ for (a) $0^{0} \leq \theta \leq 12^{0}$ and (b) $12^{0} \leq \theta \leq 30^{\circ}\left(\Delta \theta=1^{0}\right)$; inset: enlarged high field, $\mathrm{T}=1.85 \mathrm{~K}$ (see text) isotherms. Arrows indicate the direction of increasing $\theta$. 


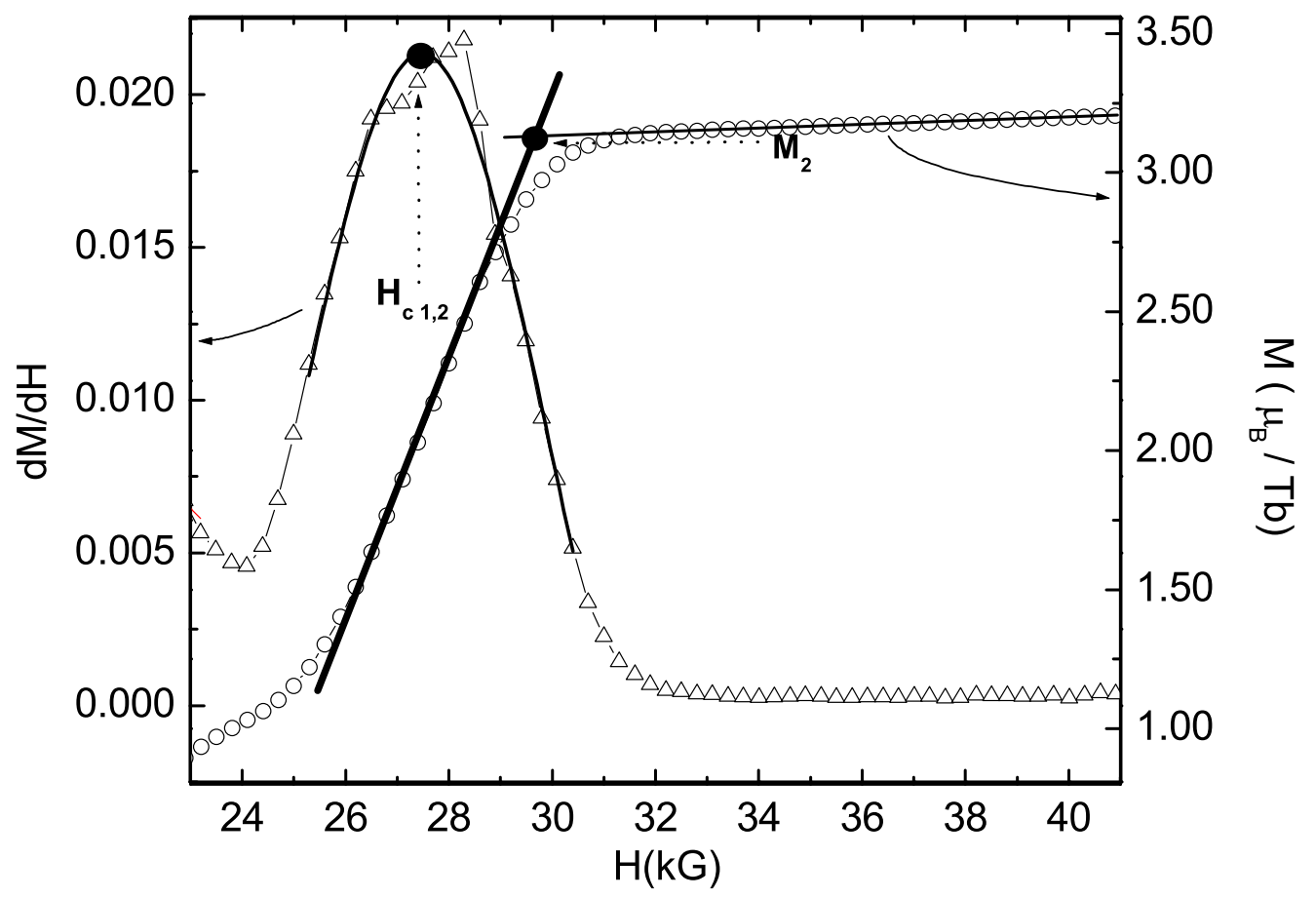

FIG. 12: Enlarged $\mathrm{M}\left(\mathrm{H} ; \theta=12^{0}\right)$ plot, and the corresponding derivative, illustrating the criteria used in determining the points in Fig.13: large dot on the $\mathrm{dM} / \mathrm{dH}$ plot indicates the critical field value, determined by the maximum of the Lorentzian fit (solid line) of the peak; straight lines on the $\mathrm{M}(\mathrm{H})$ plot are fits to the magnetization plateau (thin), extrapolated down to intersect the maximum-slope line (thick), giving the $M_{2}$ value (large dot). Note: the quality of the Lorentzian fit is representative of some of the poorer fits. 

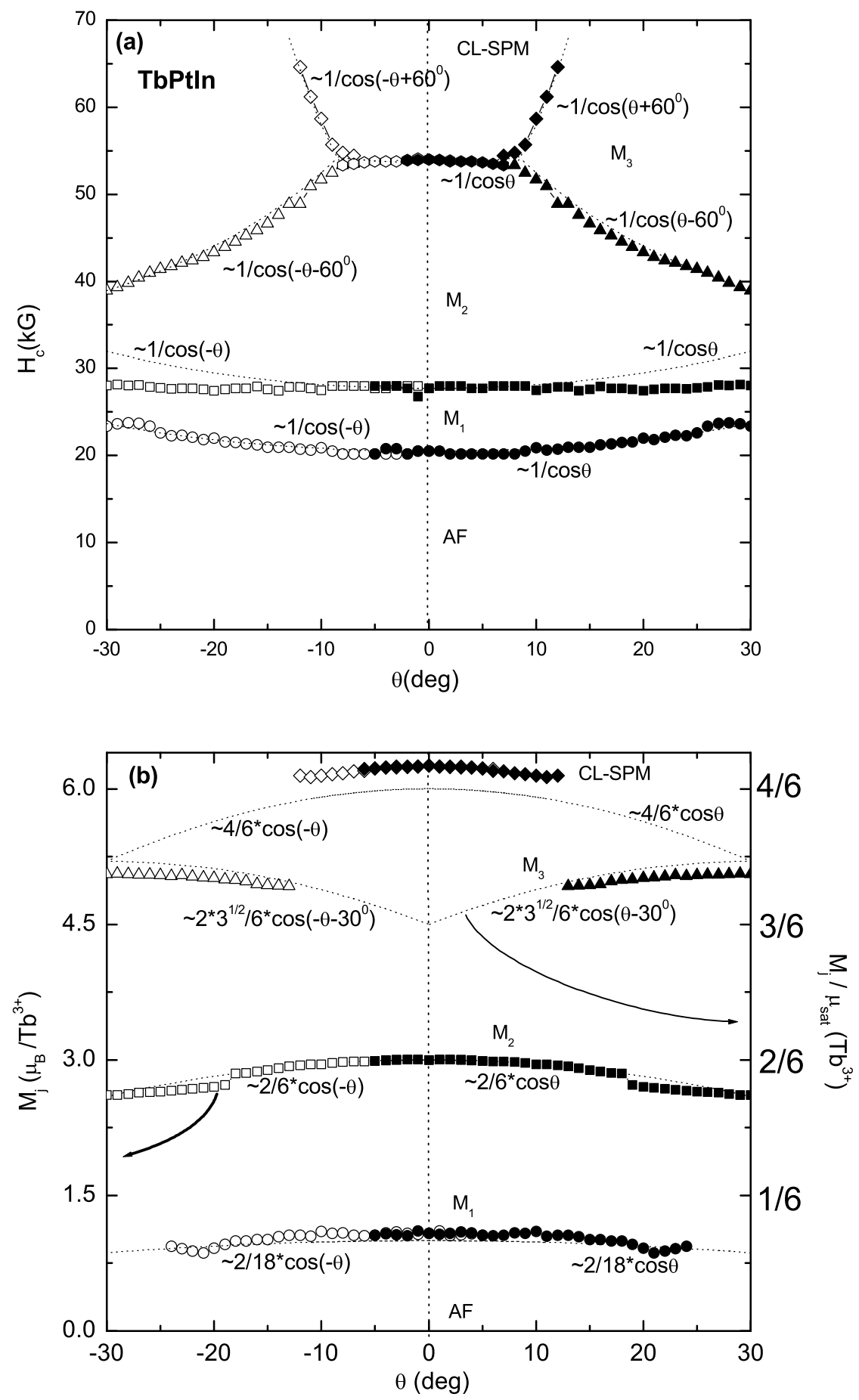

FIG. 13: (a) Measured critical fields $\mathrm{H}_{c i, j}$ and (b) locally saturated magnetizations $\mathrm{M}_{j}$ (full symbols) as a function of angle $\theta$ measured from the [120] direction. Open symbols are reflections of the measured data across the $\theta=0^{0}$ direction. Also shown are the calculated angular dependencies (dotted lines). 


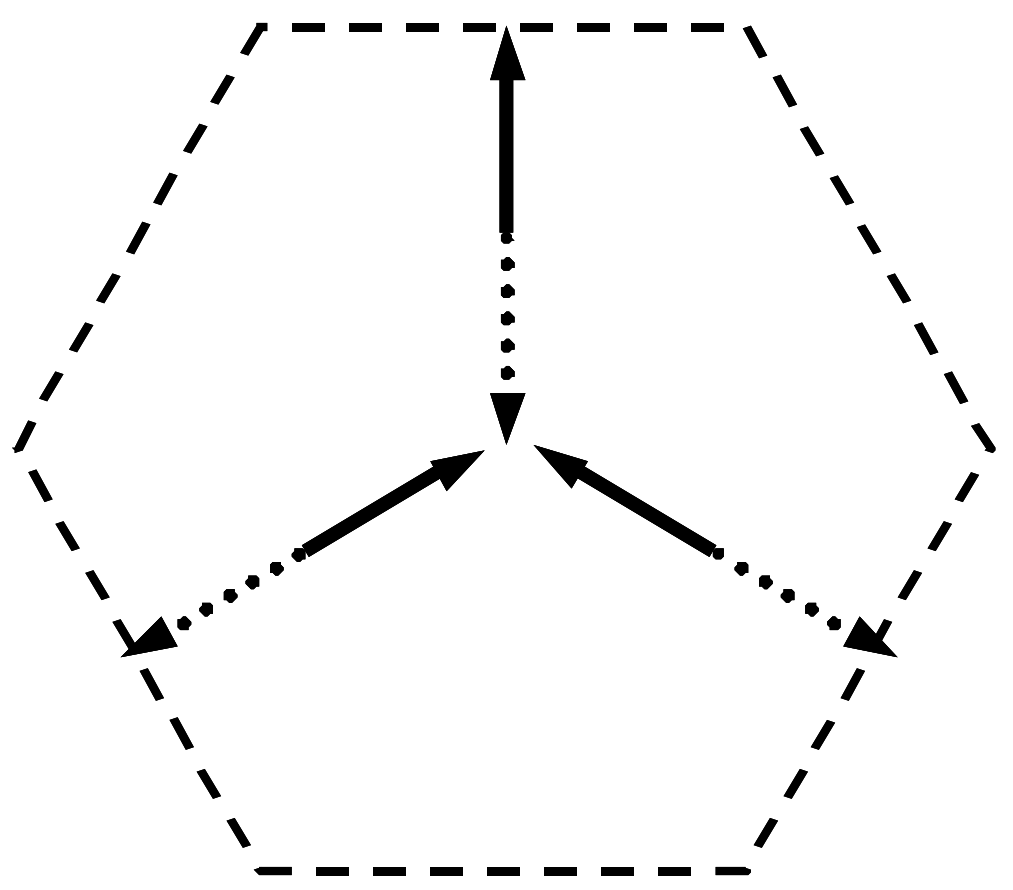

FIG. 14: Schematic representation of the three Ising-like systems model (with three distinct R in the unit cell, sitting in unique orthorhombic sites): solid arrows- 'up', and dotted arrows- 'down' positions of the magnetic moments along the easy axes. 


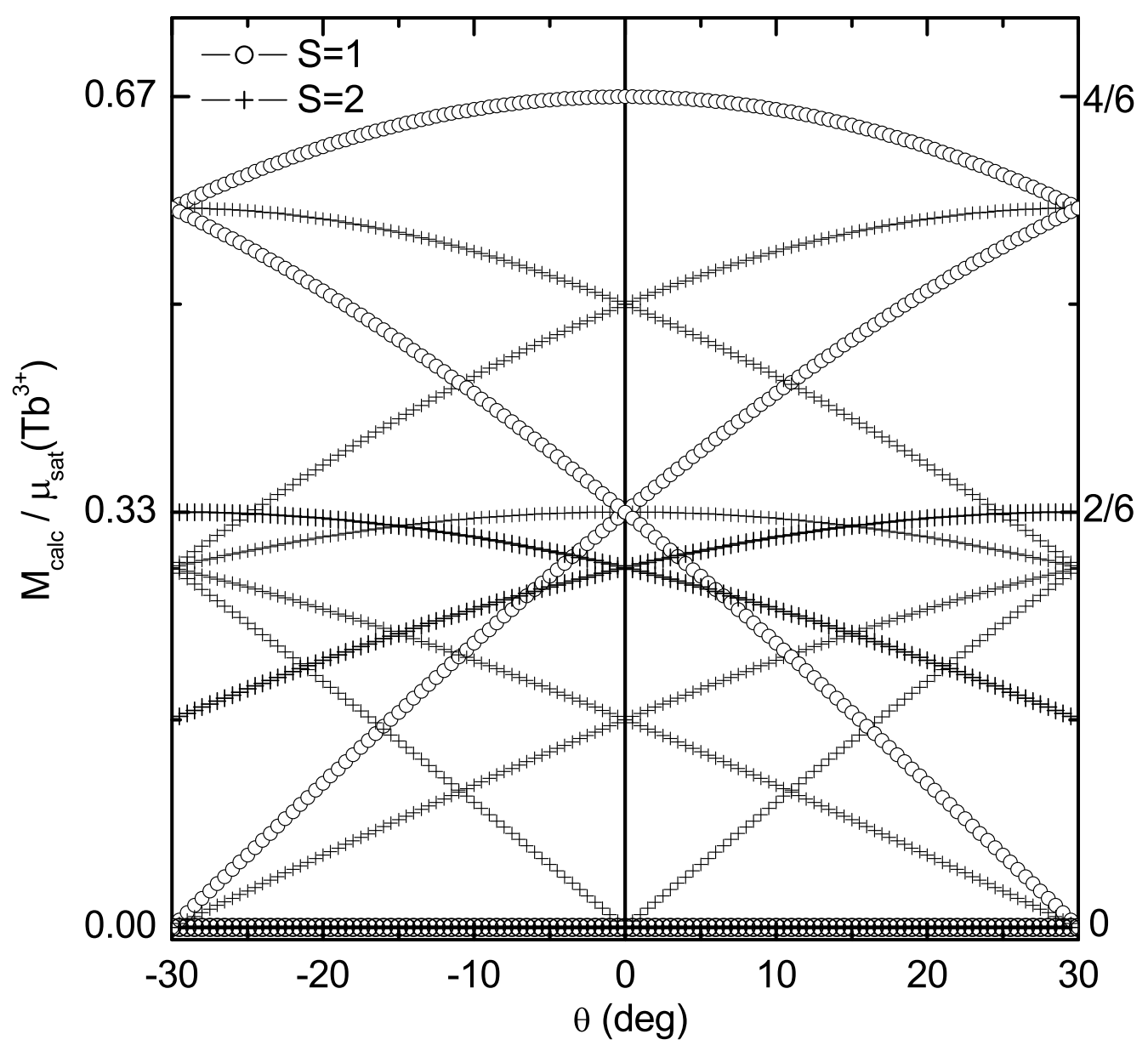

FIG. 15: Calculated magnetizations as a function of $\theta$, based on the three coplanar Ising model: open circles - S = 1 and crosses- $\mathrm{S}=2$ (see text for details). 


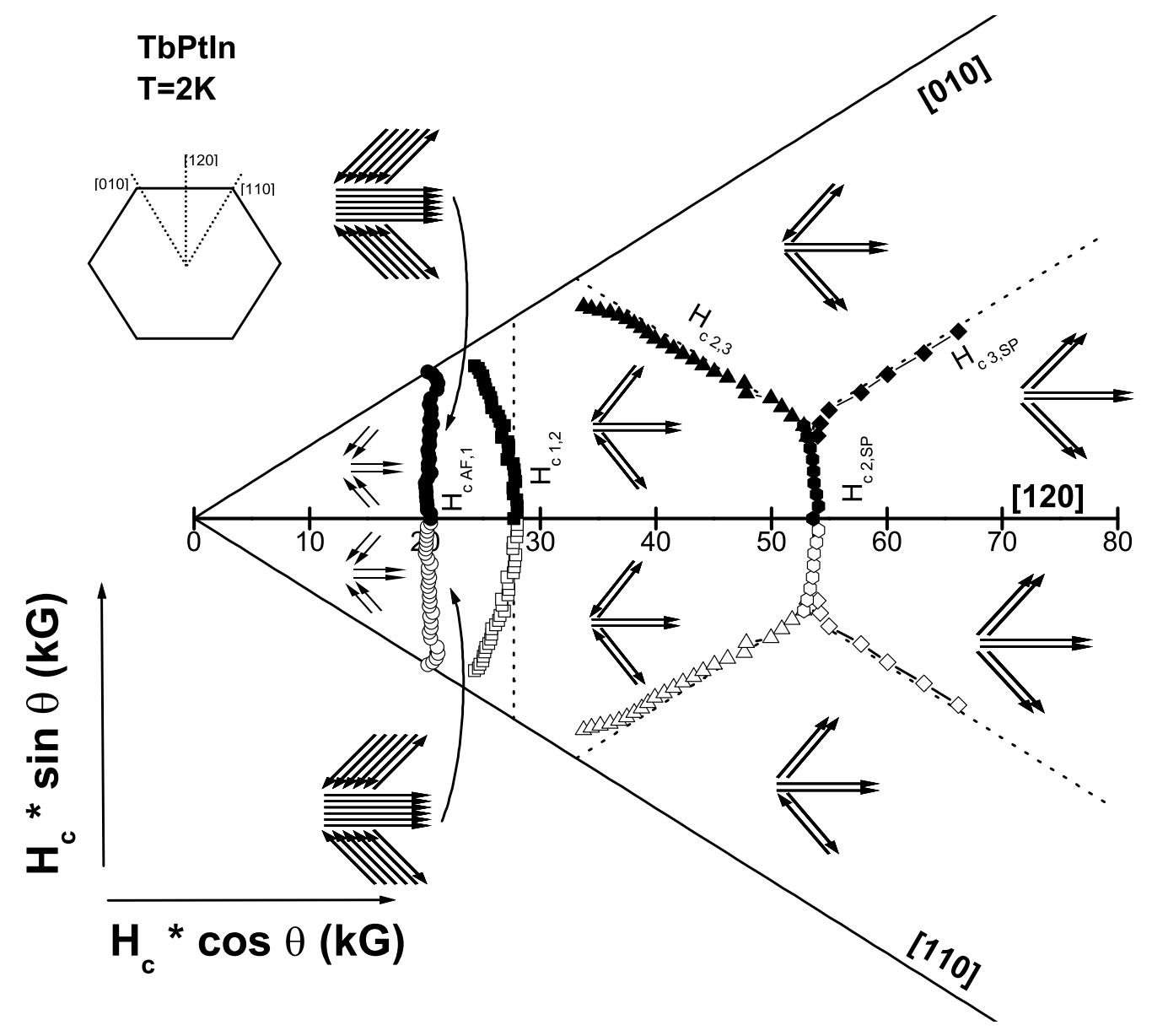

FIG. 16: Polar plot of the critical fields $\mathrm{H}_{c i, j}$, with one possible moment configuration shown for each observed metamagnetic state; open symbols represent reflections of the measured data -full symbols- across the $\theta=0^{0}$ direction (see text). 


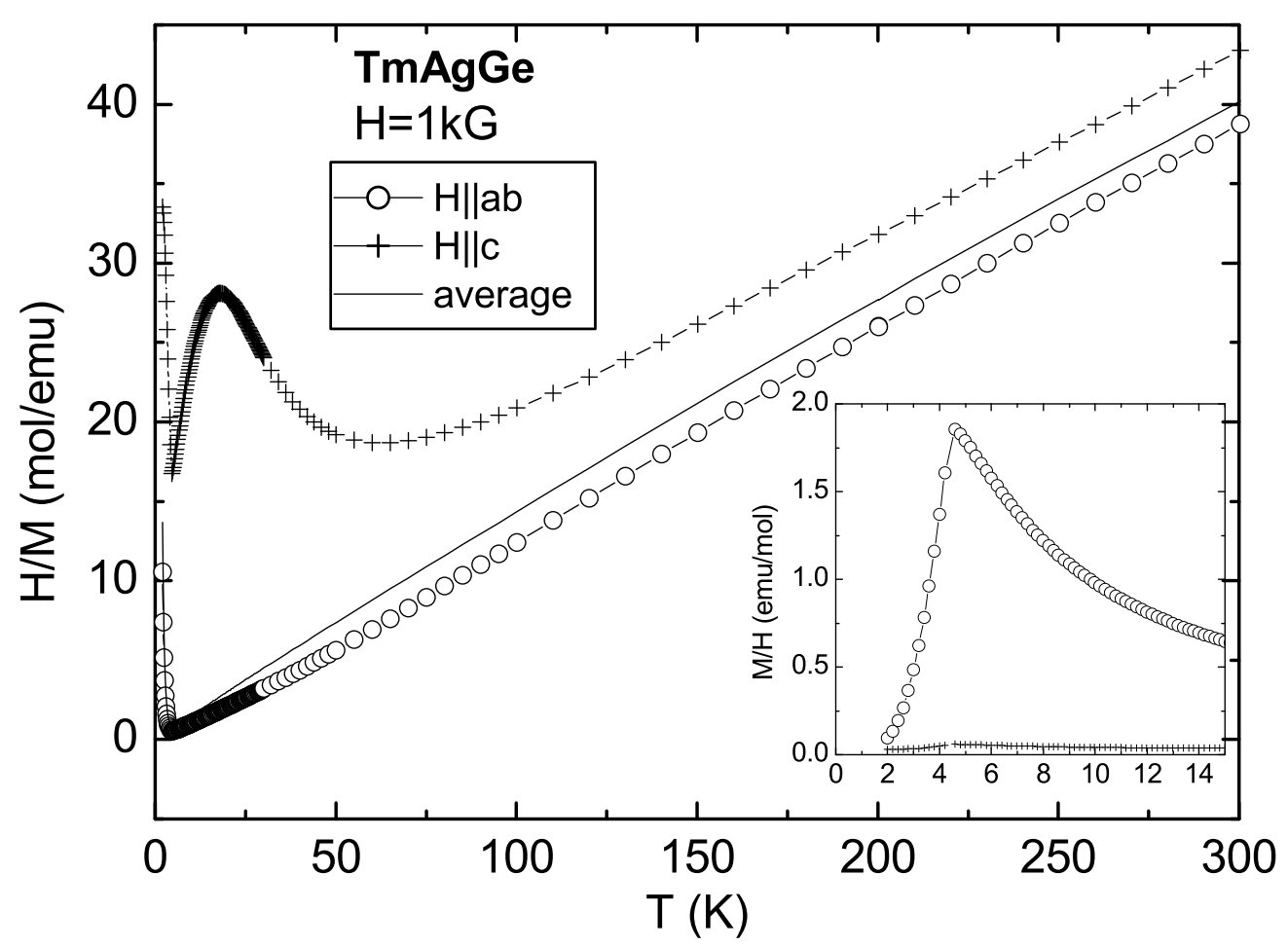

FIG. 17: Anisotropic inverse susceptibility of TmAgGe (symbols) and the calculated polycrystalline average (line); inset: low-temperature anisotropic susceptibilities. 


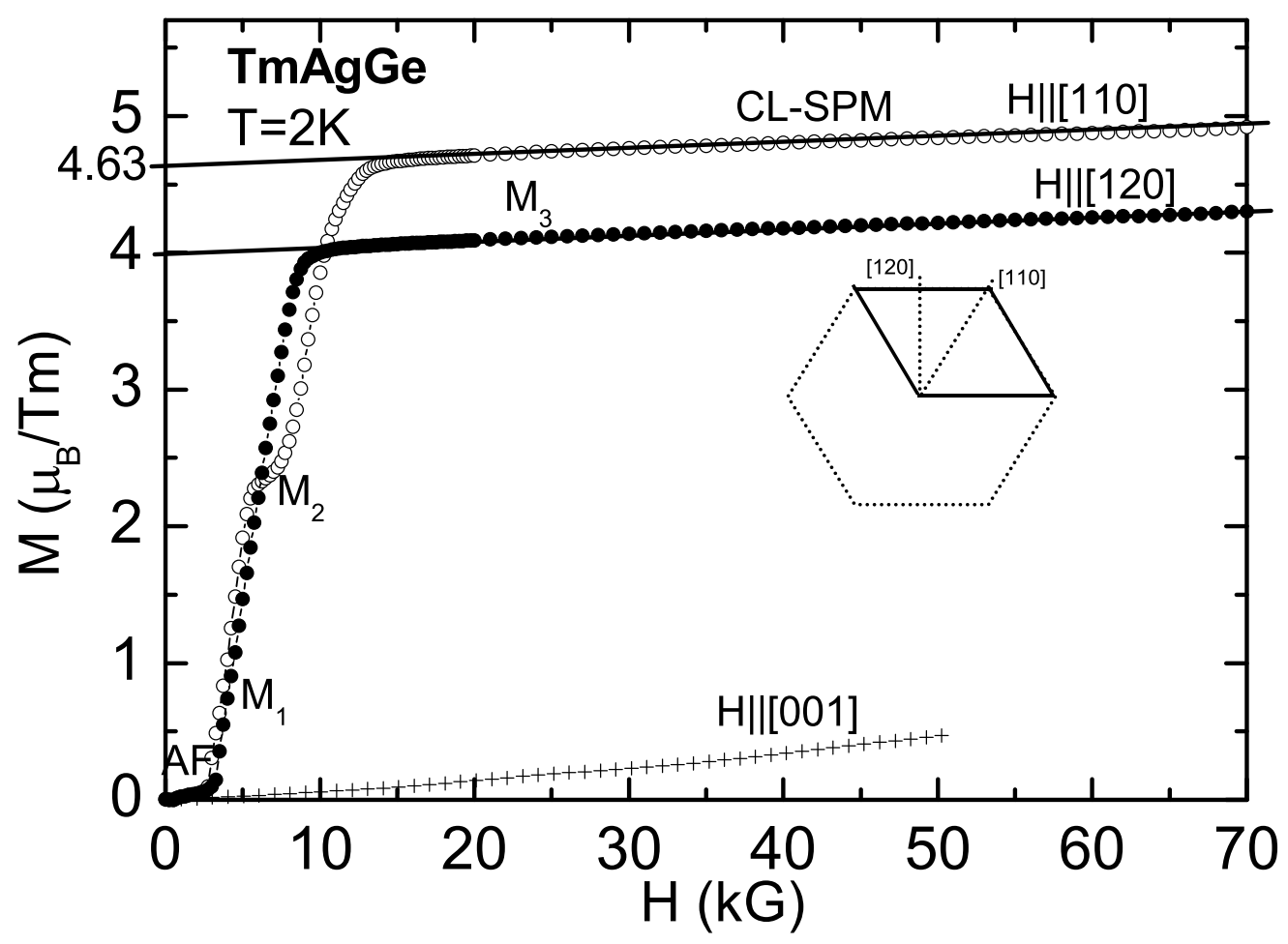

FIG. 18: Anisotropic field-dependent magnetization, with the solid lines being the linear fits of the high-field plateaus, extrapolated down to $\mathrm{H}=0$ (see text). 


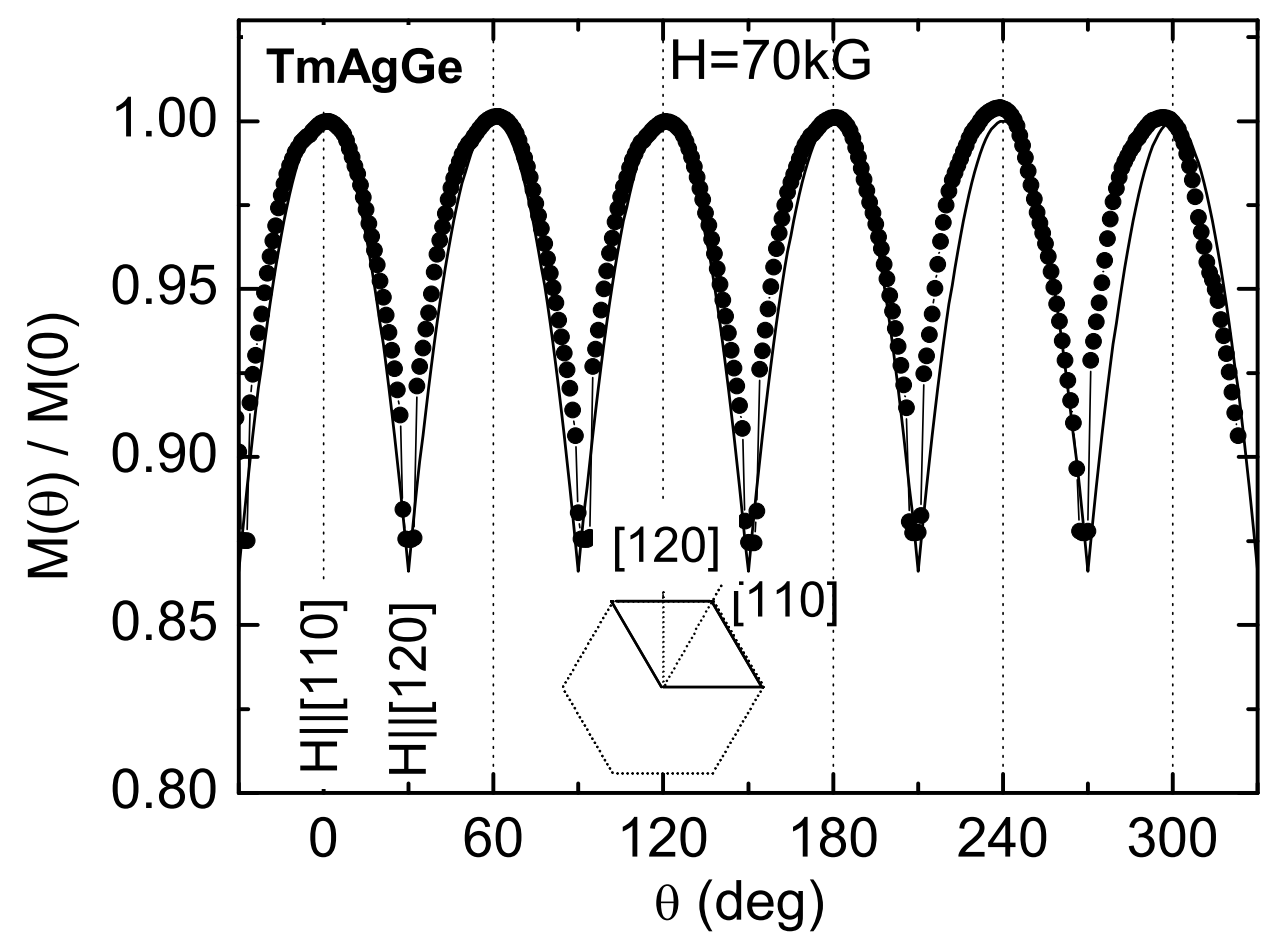

FIG. 19: $\mathrm{M}(\theta)$ of TmAgGe (full symbols) at $\mathrm{T}=2 \mathrm{~K}$ and $\mathrm{H}$ $=70 \mathrm{kG}, \mathrm{H} \perp \mathrm{c}$. Solid line: calculated magnetization $\mathrm{M}_{\max } *$ $\cos \left(\theta-n * 60^{\circ}\right), n$-integer. Note: $\theta=0^{0}$ is defined at the [110] direction. 

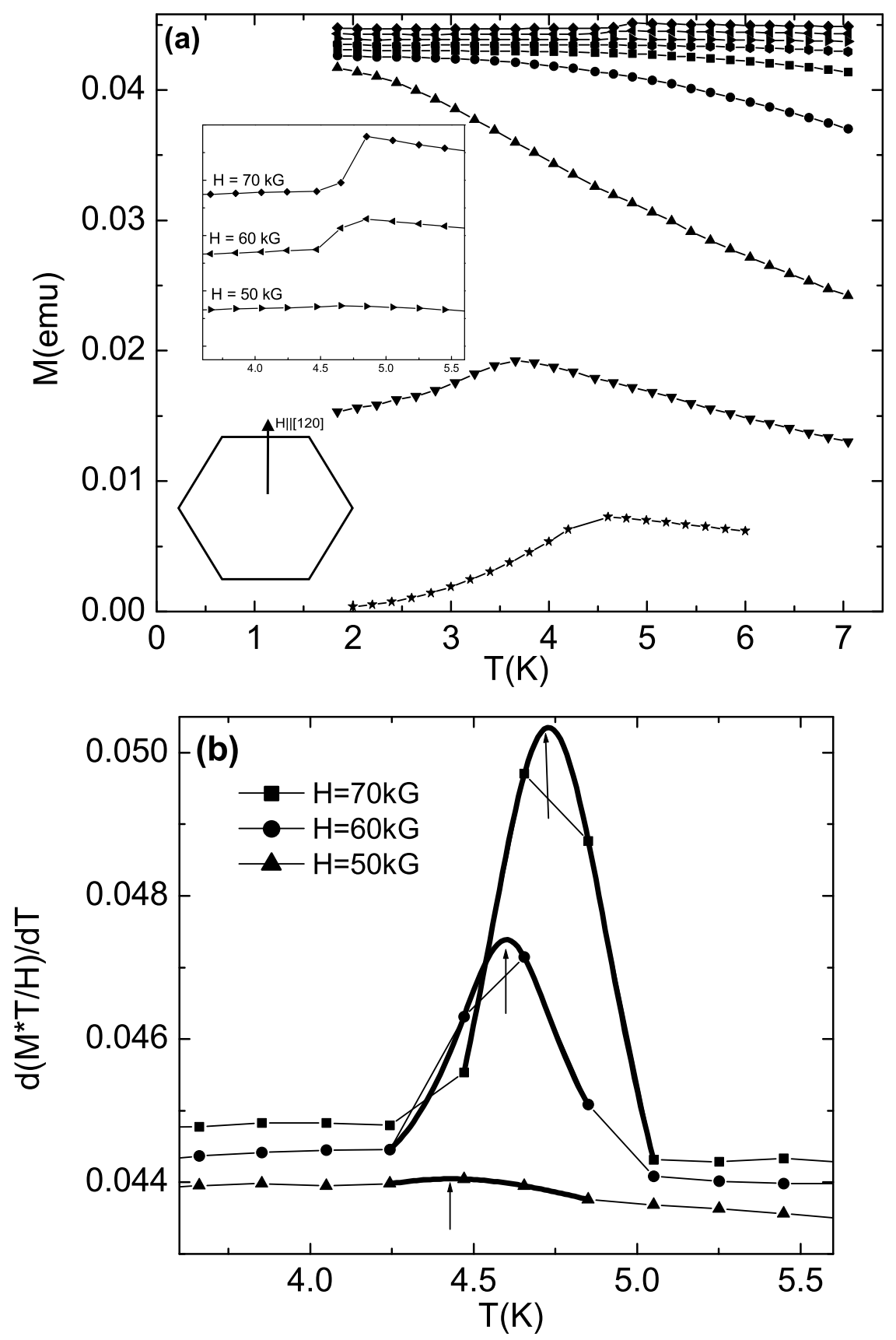

FIG. 20: (a) $\mathrm{M}(\mathrm{T})$ data for $\mathrm{H}=1,5 \mathrm{kG}$ and $10-70 \mathrm{kG}$ $(\Delta \mathrm{H}=10 \mathrm{kG})$ for $\mathrm{H} \|[120]$, with enlarged high-field data in the inset; (b) $\mathrm{M}^{*} \mathrm{~T} / \mathrm{H}$ derivatives for $\mathrm{H}=50,60$ and $70 \mathrm{kG}$, together with the Lorentzian fits of the maxima (solid lines), illustrating how the vertical line in Fig.22c was determined for high applied fields. 

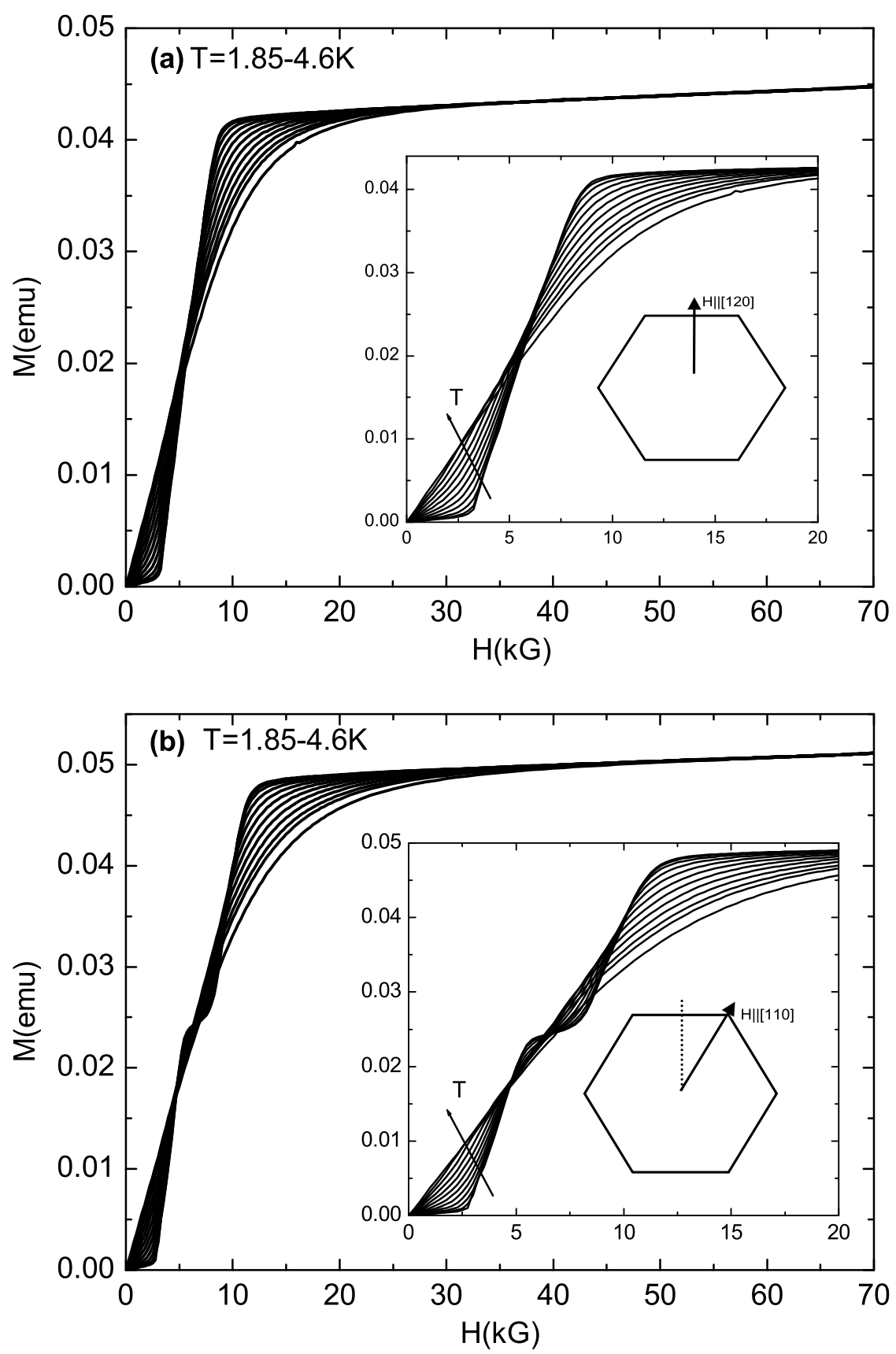

FIG. 21: $\mathrm{M}(\mathrm{H})$ isotherms for $\mathrm{T}=1.85,2-4 \mathrm{~K}(\Delta T=0.25$ $\mathrm{K}), 4.2$ and $4.6 \mathrm{~K}$ for (a) $\mathrm{H} \|[120]$ and (b) $\mathrm{H} \|[110]$; insets show the enlarged data around the metamagnetic transitions. (Arrows indicate direction of increasing T.) 


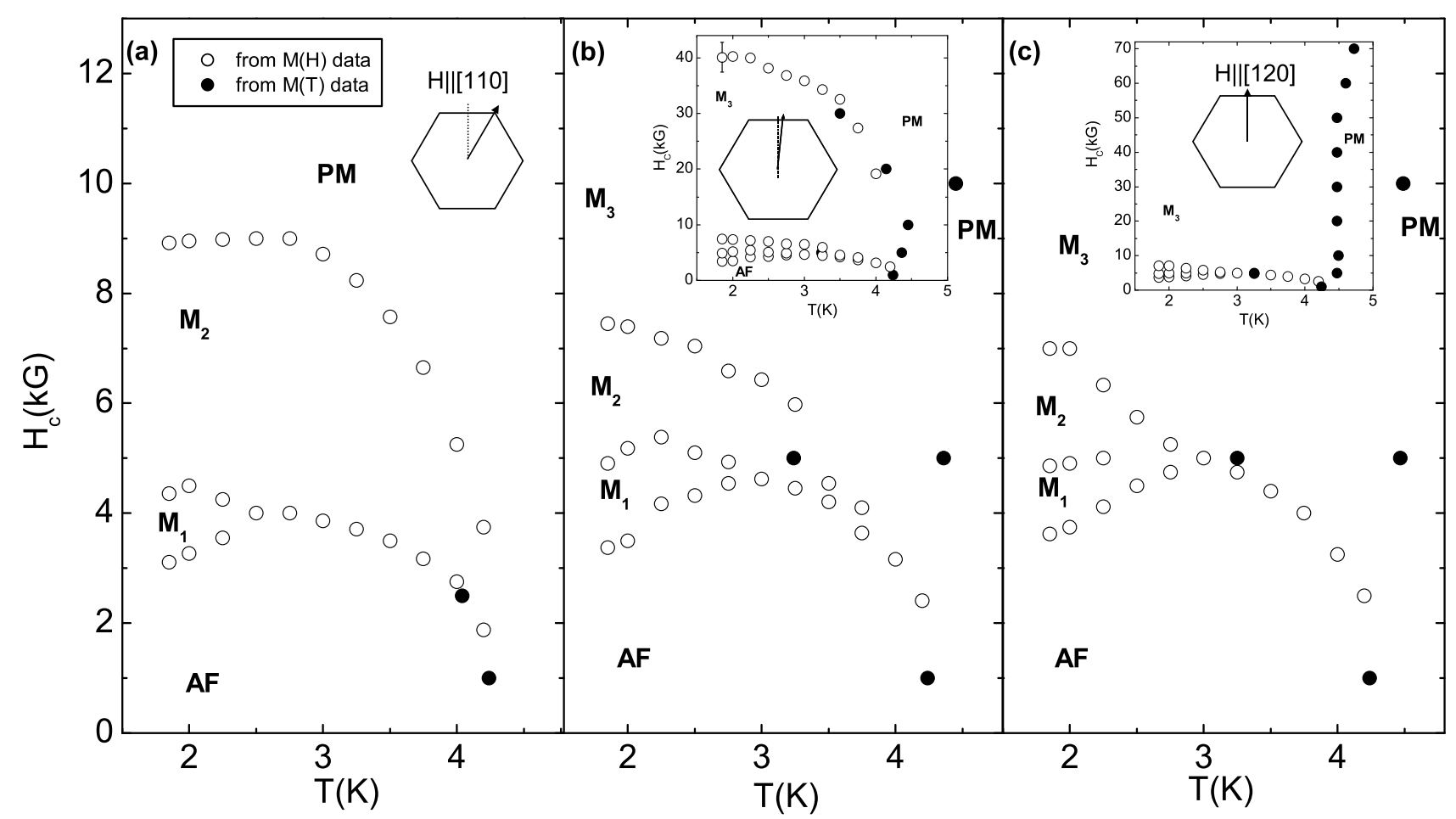

FIG. 22: H-T phase diagrams for TmAgGe, with (a) H\|[110] and (c) $\mathrm{H} \|[120]$, as determined from magnetization data in Fig.20-21; (b) intermediate-position phase diagram (see text); the error bar shown in inset of (b) represents the range of values for the high-field line, as determined from Fig.23. 


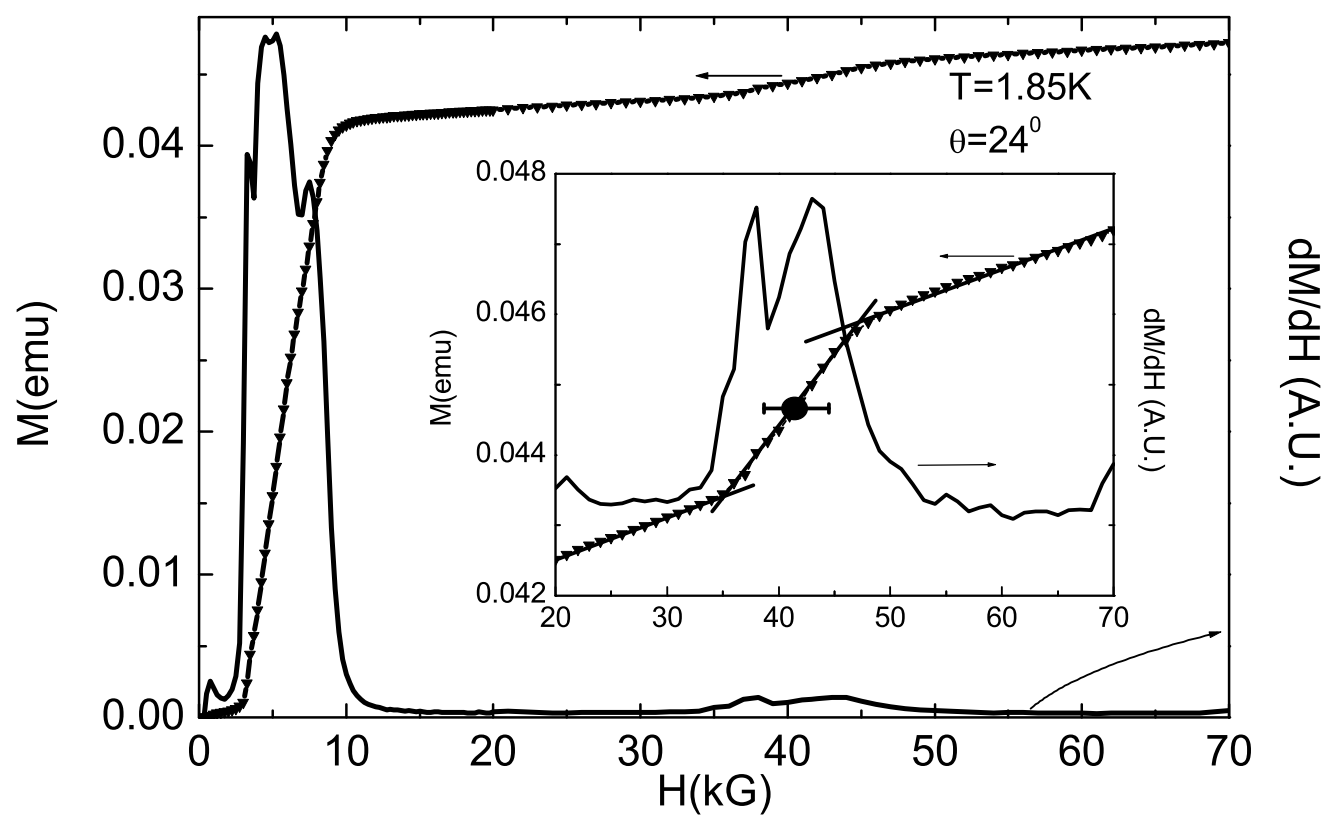

FIG. 23: $\mathrm{M}(\mathrm{H})$ for $\mathrm{T}=1.85 \mathrm{~K}$ (symbols), and the corresponding derivative (line). The inset shows an enlargement around the high field transition, to exemplify the two criteria used for determining this critical field (see text). Note: the error bar shown in the inset (determined from the position of the peaks in the derivative) gives a caliper of the uncertainty in determining this critical field value. 

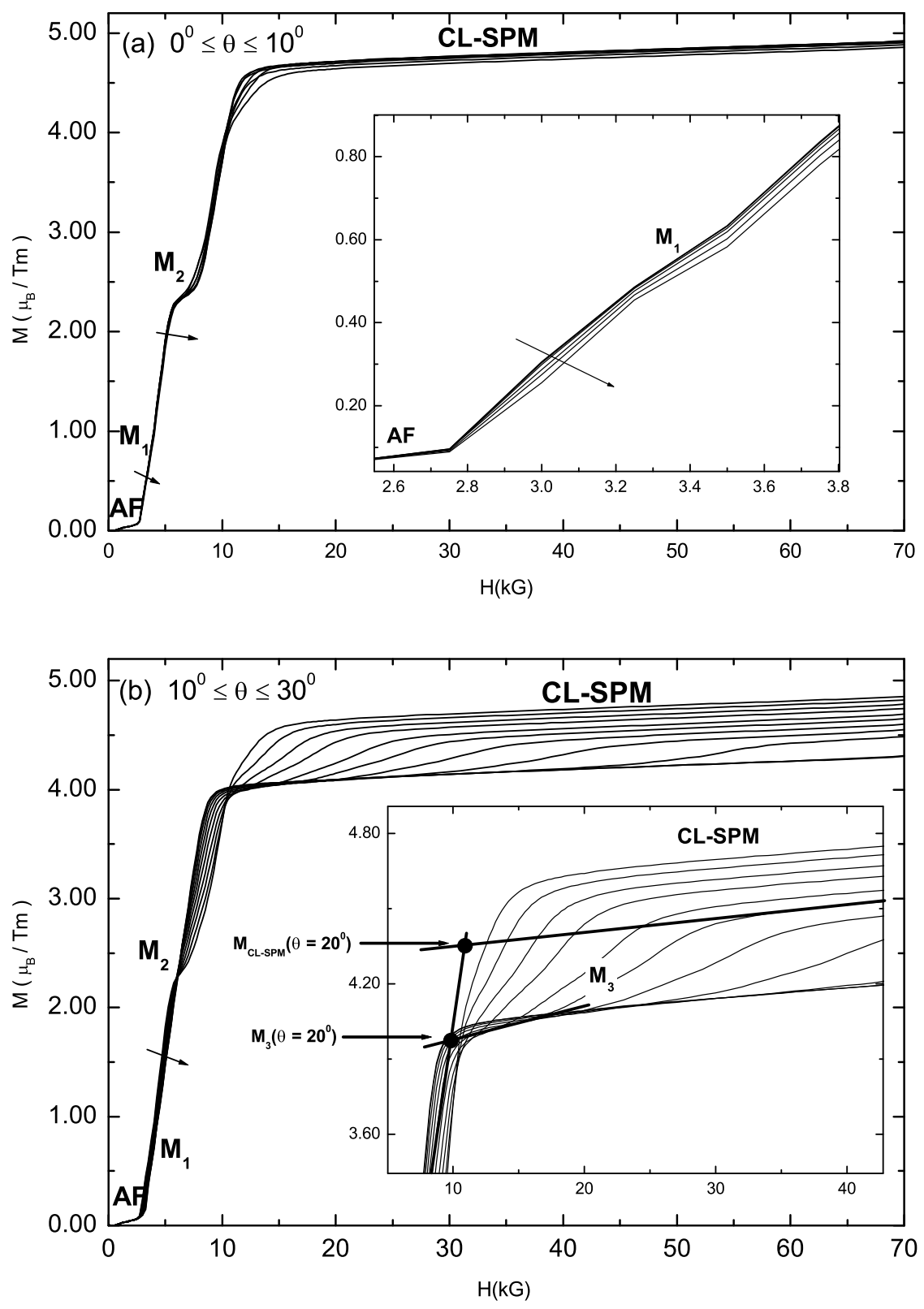

FIG. 24: $\mathrm{M}(\mathrm{H})$ isotherms $(T=2.0 \mathrm{~K})$ for (a) $0^{0} \leq \theta \leq 10^{\circ}$, $\Delta \theta=2^{0}$ (enlarged $\mathrm{M}_{1}$ state shown in the inset) and (b) $10^{0} \leq \theta \leq 30^{\circ}, \Delta \theta=2^{0}$; inset in (b) shows the extrapolation of the two higher metamagnetic states down to lower fields, such that the intersection with the maximum-slope line gives the magnetization values $\mathrm{M}_{3}$ and $\mathrm{M}_{C L-S P M}$ - solid dots (see text). Arrows indicate increasing $\theta$. 

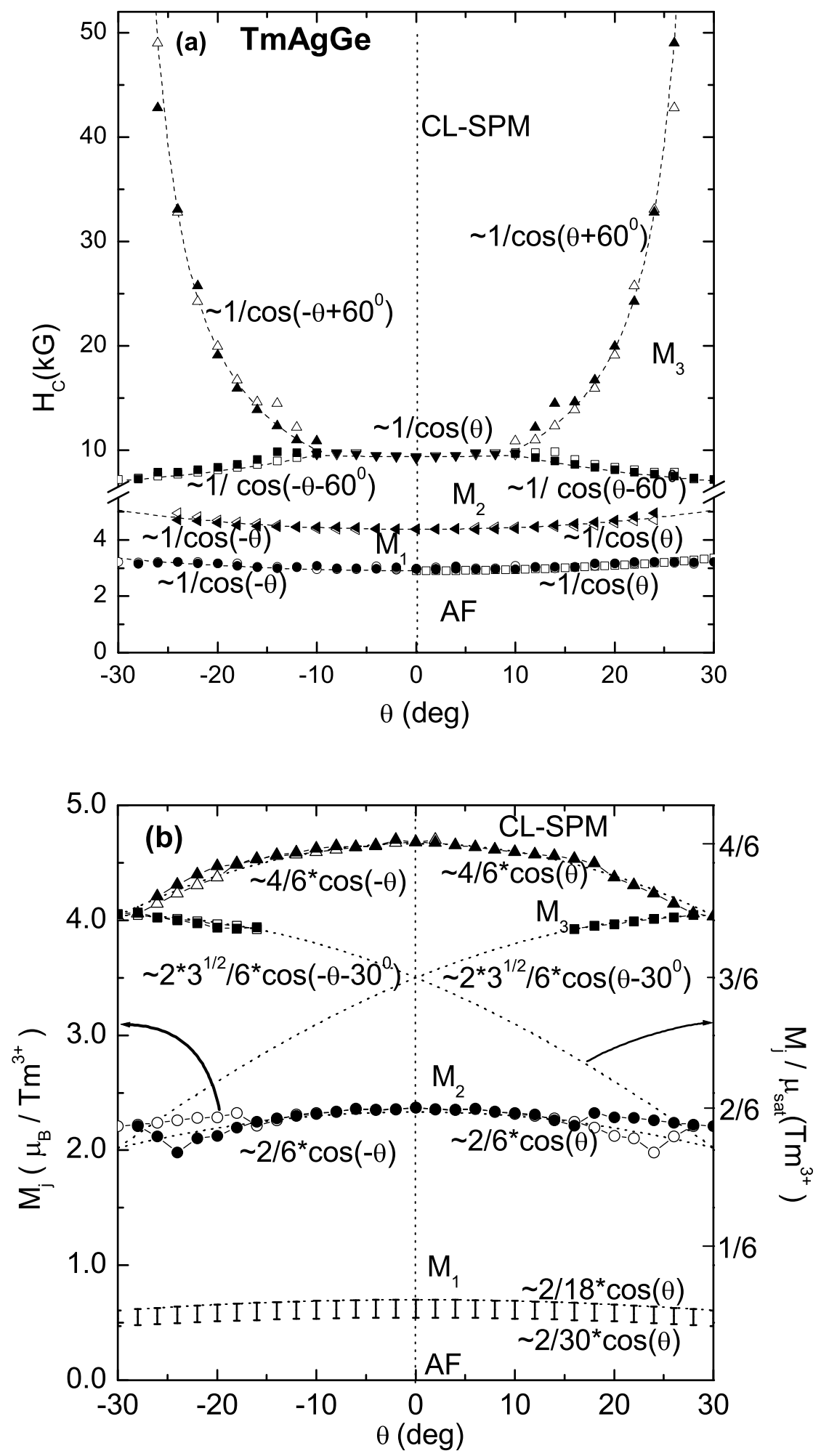

FIG. 25: (a) Measured critical fields $H_{c i, j}$ and (b) locally saturated magnetizations $M_{j}$ (full symbols), as a function of angle $\theta$ measured from the easy axis. Open symbols are reflections across the $\theta=0^{0}$ direction (see text). Also shown are the calculated angular dependencies of $H_{c i, j}$ and $M_{j}$ (dotted lines). The error bars shown in the low part of (b) give the range of values that we can infer for $M_{1}$ from the data shown in Fig.24. 


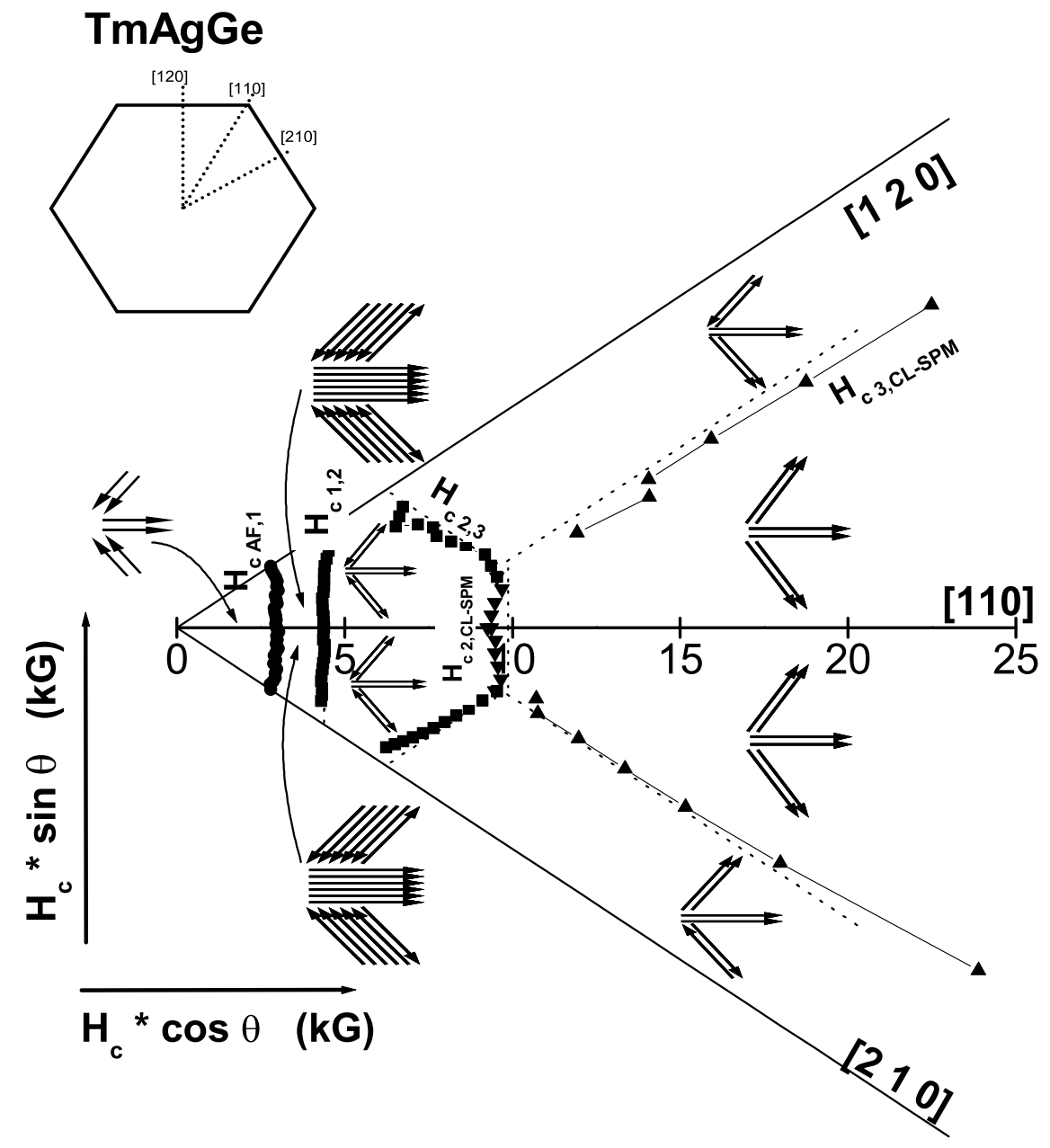

FIG. 26: Polar plot of the critical fields $H_{c}$, with one of the possible moment configurations shown for each observed metamagnetic state. 

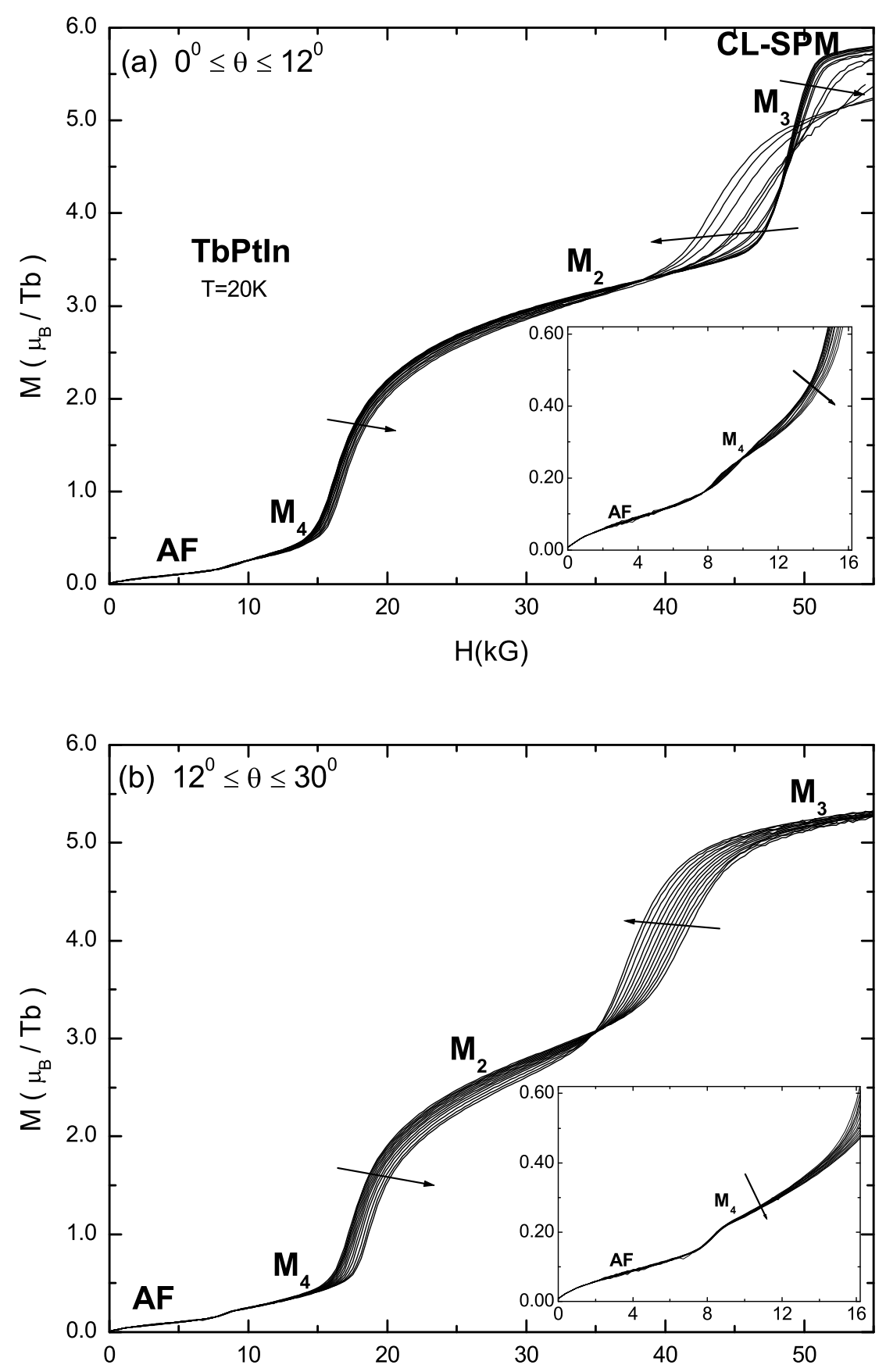

FIG. 27: $\mathrm{M}(\mathrm{H})$ isotherms at $T=20 \mathrm{~K}$ for (a) $0^{0} \leq \theta \leq 12^{0}$ and (b) $12^{0} \leq \theta \leq 30^{\circ}\left(\Delta \theta=1^{0}\right)$; inset: enlarged $M_{4}$ state. Arrows indicate the direction of increasing $\theta$. 

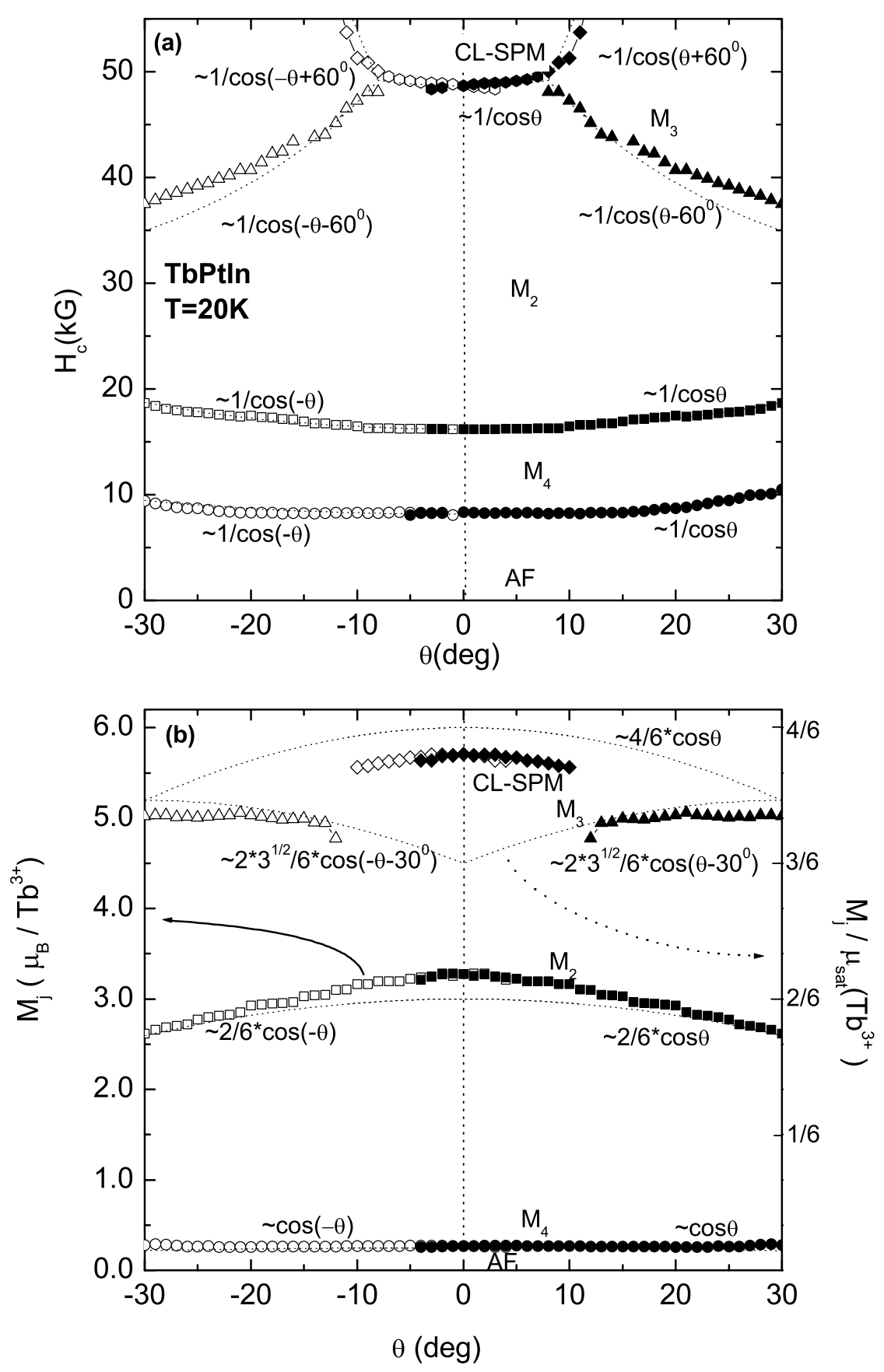

FIG. 28: (a) Measured critical fields $H_{c i, j}$ and (b) locally saturated magnetizations $M_{j}$ (full symbols), as a function of angle $\theta$, for $T=20 \mathrm{~K}$. Open symbols are reflections across the $\theta=0^{0}$ direction. Also shown are the calculated angular dependencies of $H_{c i, j}$ and $M_{j}$ (dotted lines). 


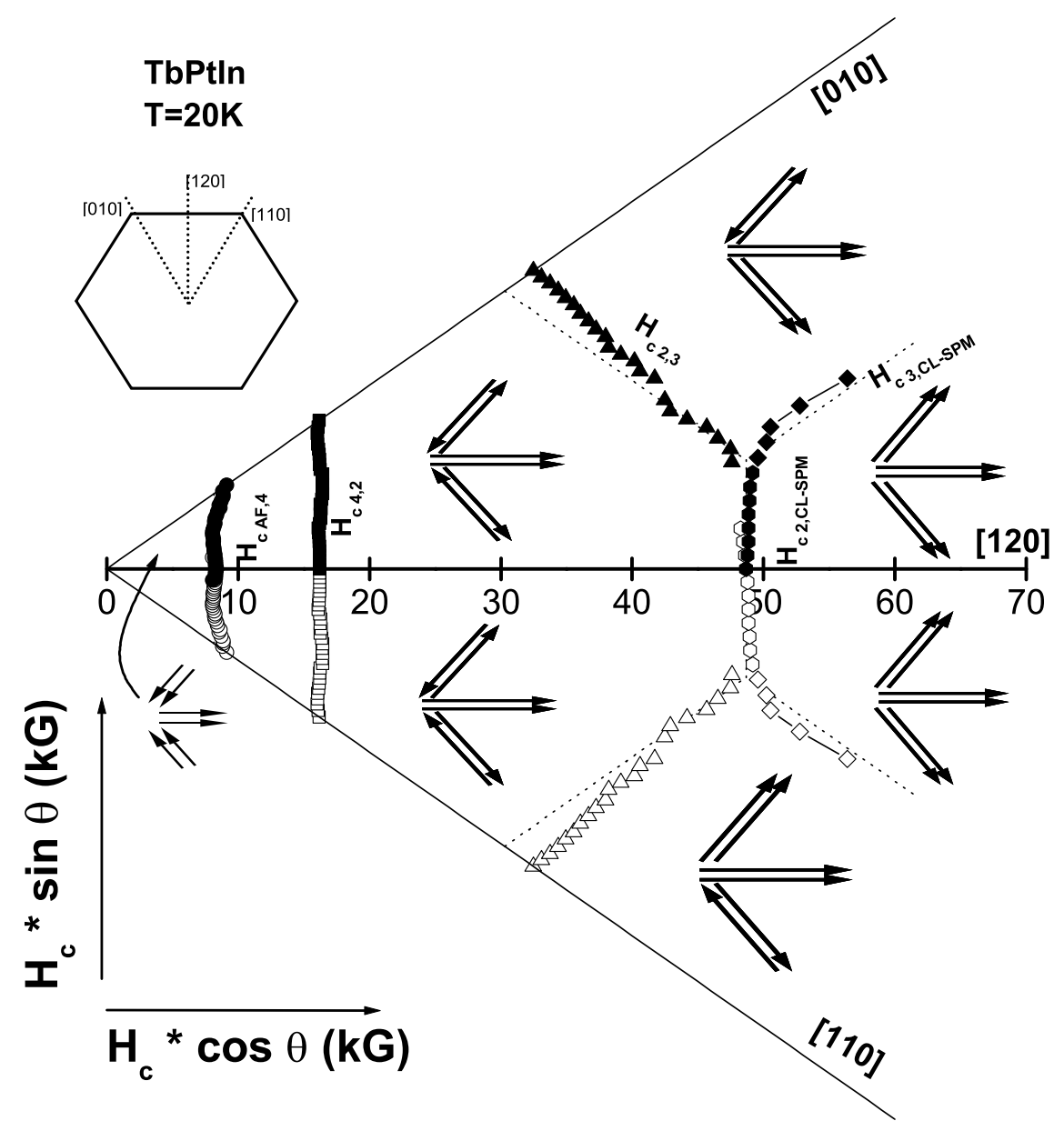

FIG. 29: Polar plot of the critical fields $H_{c i, j}$, with one possible moment configuration shown for each observed metamagnetic state (except for $M_{1}$, where the moment configuration is uncertain- see text); open symbols represent reflections of the measured data-full symbols- across the $\theta=0^{0}$ direction. 
(a)
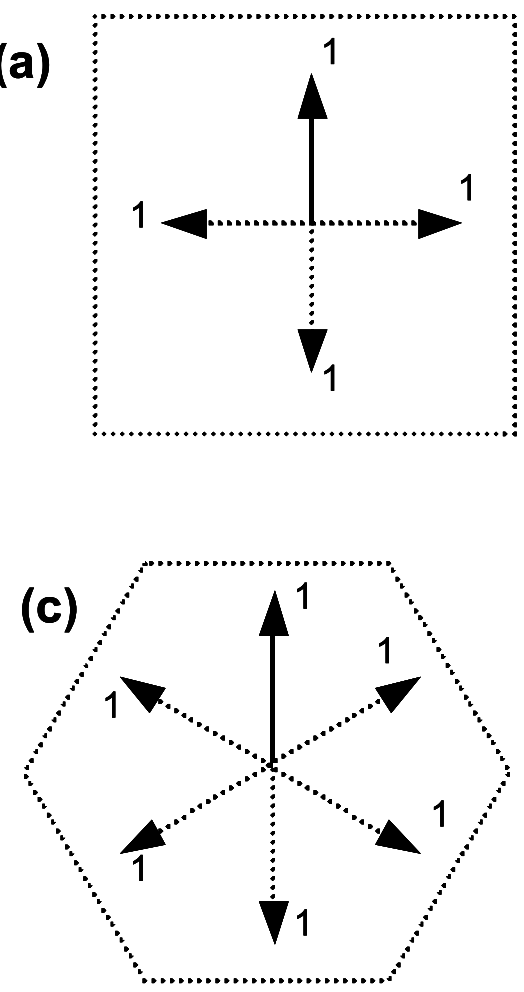
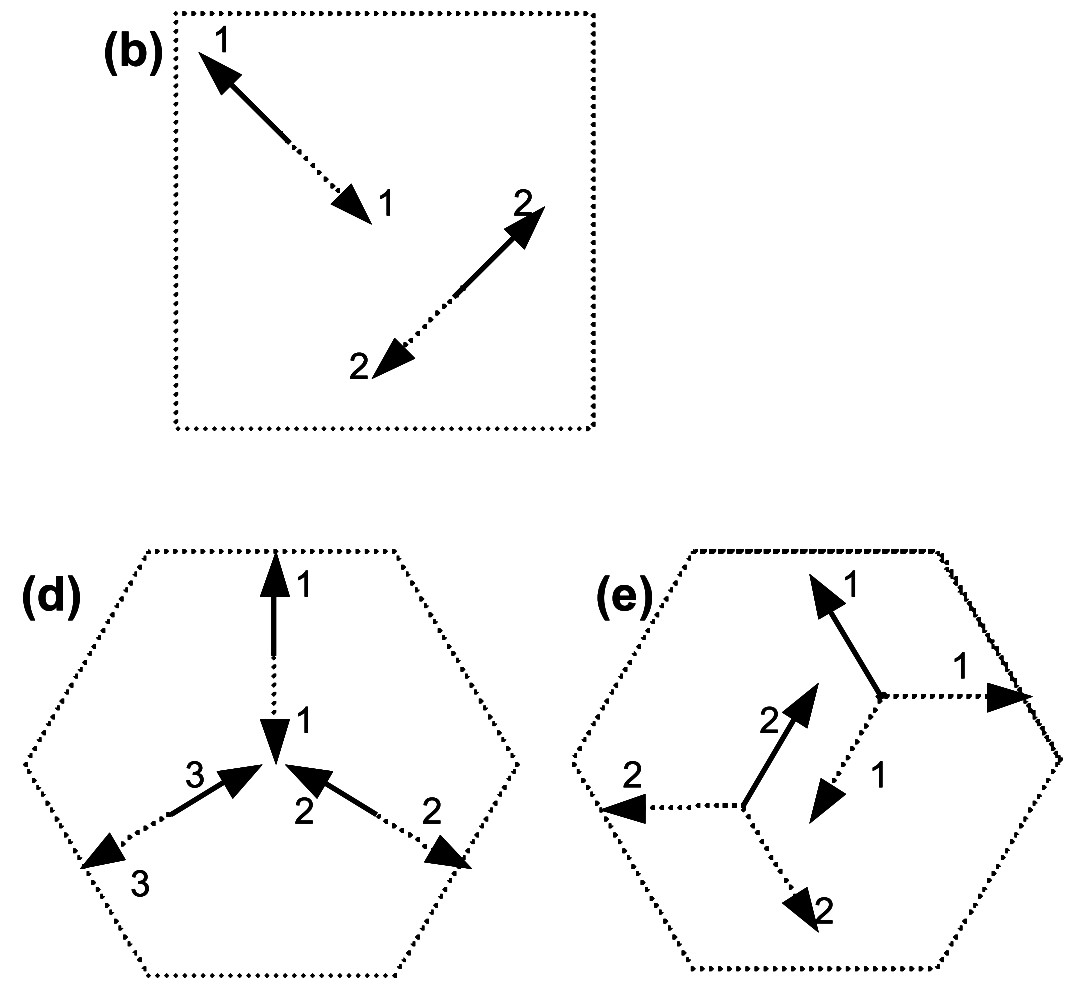

FIG. 30: Plausible models for extremely anisotropic, planar compounds (see text) with (a-b) tetragonal and (c-e) hexagonal unit cells. The numbers are used to identify the different magnetic moments in the unit cell. (a) Four position clock model describing tetragonal systems with one $\mathrm{R}$ in tetragonal point symmetry; (b) Double coplanar Ising-like model: for orthorhombic point symmetry in tetragonal unit cell, two magnetic moments would be necessary (Ising-like systems, $90^{\circ}$ away from each other in the basal plane). (c) Six position clock model: one magnetic moment with six possible orientations (arrows along six high symmetry orientations in the basal plane); (d) Triple coplanar Ising-like model: three $\mathrm{R}$ ions in unique orthorhombic point symmetry are needed in a hexagonal compound (three Ising-like systems, $60^{\circ}$ away from each other in the basal plane); (e) A double coplanar three position clock model can describe hexagonal systems with two magnetic moments in unique trigonal point symmetry position, with three possible orientations $\left(120^{\circ}\right.$ away from each other in the basal plane) for each. In all cases, the corresponding CL-SPM states (described in text) are represented by full arrows (applied field is assumed to be vertically up. 